\title{
Joint Optimization of Transmit Power-Time and Bit Energy Efficiency in CDMA Wireless Sensor Networks ${ }^{1}$
}

\author{
Tao Shu, Marwan Krunz, and Sarma Vrudhula \\ Department of Electrical and Computer Engineering \\ University of Arizona \\ Tucson, AZ 85721, USA \\ Email: $\{$ tshu, krunz, vrudhula\}@ece.arizona.edu \\ Technical Report \\ TR-UA-ECE-2005-3
}

April 14, 2005

\footnotetext{
${ }^{1}$ This work was supported in part by the National Science Foundation through grants ANI-0095626, ANI0313234, and ANI-0325979; and in part by the Center for Low Power Electronics (CLPE) at the University of Arizona. CLPE is supported by NSF (grant EEC-9523338), the State of Arizona, and a consortium of industrial partners. Any opinions, findings, and conclusions or recommendations expressed in this material are those of the author(s) and do not necessarily reflect the views of the National Science Foundation.
} 


\begin{abstract}
In this paper, we address the problem of minimizing energy consumption in a CDMA wireless sensor network (WSN), where multiple sensor nodes transmit data simultaneously to a common remote sink. A comprehensive energy consumption model is proposed, which accounts for both the transmit and circuit energy. Energy consumption is minimized by jointly optimizing the transmit power and transmission time for each active node in the network. The optimization problem is formulated as a non-convex optimization. Numerical as well as closed-form approximate analytical solutions are provided. For the numerical solution, we show that the formulation can be transformed into a convex geometric programming (GP), for which fast algorithms, such as Interior Point Method, can be applied. For the closed-form solution, we prove that the joint power/time optimization can be decoupled into two sequential sub-problems: optimization of transmit power with transmission time serving as a parameter, and then optimization of the transmission time. We show that the first sub-problem is a linear programming while the second one can be well approximated as a convex programming problem. Accordingly, closed-form solutions are found for both sub-problems, and hence for the original formulation. Taking advantage of these analytical results, we further derive the bit energy efficiency (BEE) performance for CDMA WSNs. Our results are verified through numerical examples and simulations.
\end{abstract}

Keywords: CDMA, sensor network, joint power and time optimization, geometric programming, convex optimization. 


\section{Introduction}

Advances in mixed-signal design and microelectronic fabrication have made it possible to integrate analog and digital processing, sensing, and wireless communication into a single integrated circuit. When packaged with a battery and other electronics, such a circuit forms a small, low cost sensor unit that can be easily deployed in large numbers to form a wireless sensor network (WSN). In the near future, it is expected that WSNs will be utilized in a wide range of military and civilian applications, such as surveillance, environment and health monitoring, inventory tracking, failure detection, and many more [1]. The individual sensors, being powered by small batteries, have very limited energy capacity. Even in moderate-size networks, the replacement of such batteries is not feasible, either due to lack of access or to prohibitive cost. Consequently, strategies for achieving very high energy efficiency so as to maximize the lifetime of the network are essential.

So far, it is known that the energy required to transmit a certain amount of information grows exponentially with the inverse of the transmission time [3]. This simple transmission power-delay tradeoff has been applied in the design of energy-efficient packet scheduling protocols for singleuser wireless links. In [4] and [5], the "lazy scheduling" approach was proposed. According to this approach, the energy used to transmit packets over a wireless link is minimized by judiciously varying packet transmission times according to the delay requirements. In [6] and [7], traffic smoothing is performed, resulting in an output packet traffic that is less bursty than the input traffic, and leading to significant power savings.

Although the tradeoff between transmission energy and transmission time has been extensively studied in the context of general wireless networks, such work is not directly applicable to WSNs due to specific features in node organization and transmission in a WSN. More specifically, because of the high density of nodes in a WSN, e.g., 20 nodes per meter ${ }^{3}$ [2], the average transmission distance between nodes is usually small. Accordingly, signal propagation tends to follow a free-space path loss model (with a path loss exponent close to 2), rather than a terrestrial propagation model (with a large loss exponent) as in a cellular environment. For such short-range transmission, the circuit energy consumption is no longer negligible relative to the transmission energy [11]. Therefore, a more complicated tradeoff emerges between energy and transmission time; although increasing the transmission time reduces the transmission energy, it also increases the circuit energy consumption. Another important feature that distinguishes a WSN from traditional wireless networks is the high correlation between nodes in a WSN. Because WSNs are often designed to cooperate on executing some joint task, less emphasis is put on per-node fairness. Accordingly, it is more reasonable to minimize the total energy consumption in the network instead of minimizing the energy consumption of individual nodes, i.e., a multi-user environment is more preferable for the optimization. Embracing the impact of circuit energy consumption and the new context of multiple access optimization, a new formulation is necessary to minimize the overall energy consumption in a WSN.

Several previous studies incorporated circuit energy in the optimization of energy consumption for a single user. In [8] circuit energy consumption was included in the analysis of a cooperative and hierarchical WSN. In [9] and [11], the authors exploit the tradeoff between transmission energy and circuit energy consumption to provide a cross-layer optimization of link-layer coding and physicallayer modulation for a single link. More recently, there has been some work on minimizing the total energy consumption in a multiple access environment. Reference [12] improves upon the work in [9]-[11] by extending the point-to-point joint energy minimization to a multiple access scenario and presenting a variable-length Time Division Multiple Access (TDMA) scheme that minimizes the total energy consumption in the network. However, two major difficulties appear when implementing the ideas in [12], namely, the need for strict synchronization between different nodes and the scalability 
of the variable-length time slot allocation approach, especially in a dense network such as a WSN.

In this paper, we present a novel formulation and a solution to the problem of energy minimization in a CDMA-based WSN, where multiple sensors are allowed to transmit data simultaneously to a remote sink. The assumptions on time synchronization and variable-time slot allocation in [12] are not imposed. The problem is formulated as the minimization of the total consumed energy subject to contraints on the received signal quality, transmission delays, and transmission powers. Both transmission energy and circuit energy consumptions are accounted for in the optimization. For a given number of information bits at each node, the minimization of energy is achieved by finding the optimal transmit power and transmission time for each sensor node.

The main contribution of this paper is twofold. First, although the objective function and the constraints in the underlying optimization problem are not convex, by exploiting the special structure of the formulation we successfully develop both numerical and closed-form analytical solutions to this problem. Numerically, this formulation is converted to a posynomial optimization problem that can be accurately solved by using geometric programming (GP). Analytically, we prove that the problem of jointly optimizing the transmission power and transmission time can be decoupled into two separate sequential sub-problems. The first is a parametric linear program for optimizing the transmission power with the transmission time being a parameter, and the second is a convex optimization problem for finding the optimal transmission time. We present closed-form solutions to both sub-problems, and consequently, to the original problem. Second, by taking advantage of the closed-form results, we further study the bit energy efficiency (BEE) for a CDMA-based WSN, defined as the minimum expected energy consumed to transmit a single information bit in the network while satisfying all constraints. For some special cases, we achieve a closed-form BEE expression and a BEE upper bound for the correlated and independent WSNs, respectively, where the amounts of data transmitted by different sensors are fully correlated or are independently and identically distributed (i.i.d.). Numerical examples and simulations are presented to validate our results. We also demonstrate the significant energy savings achieved by joint transmission power/time optimization.

The rest of this paper is organized as follows. We describe the system model in Section II. We formulate the problem and present the geometric programming-based numerical solution in Section III. Section IV presents an approximate closed-form analytical solution to the energy-minimization problem. Based on this solution, we study the BEE performance for a CDMA-based WSN in Section V. Section VI presents numerical examples and simulations, and Section VII concludes our work.

\section{Model Description}

\subsection{System Model}

We consider a DS-CDMA-based WSN [13][14] that consists of a set of densely distributed sensor nodes $\mathbf{S}$. The nodes transmit their data to a remote base station in a one-hop WSN or to a local cluster head in a clustered WSN [17]-[19]. Let $o$ denote the destination node and let $N$ be the number of active sensors at any given time instant, as illustrated in Figure 1. The information from the $N$ sensors is transmitted simultaneously over a spread-spectrum bandwidth of $W \mathrm{~Hz}$. The single-sided power spectrum density of the additive white Gaussian noise (AWGN) is $N_{0}$ watt/Hz.

Per-cycle transmission power and transmission time control for all sensor nodes is performed by $o$. For sensor $i(i=1, \ldots, N)$, there are $B_{i}$ bits in the queue waiting to be transmitted to $o$ using transmit power $P_{t i}$ and for a transmission duration $T_{i}$. Different transmission rates are supported by using variable spreading gains. Let the channel gain between nodes $i$ and $o$ be $h_{i}$ and assume the channel is stationary for the duration $T_{i}$. The quality-of-service (QoS) requirement of sensor $i$ 
is presented by the triple $\left(\gamma_{i}, T_{i}^{\text {limit }}, P_{\max }\right)$, where $\gamma_{i}$ is the minimum bit-energy-to-interference-ratio threshold for the received signal from sensor $i, T_{i}^{\text {limit }} \geq T_{i}$ is an upper limit on the transmission delay, and $P_{\max } \geq P_{t i}$ is the maximum transmit power (assumed the same for all nodes). As is common in DS-CDMA systems, we assume BPSK modulation. We must point out that, although we assume a common $P_{\max }$ for all nodes and BPSK modulation for the system, the analysis presented is not limited to these specific assumptions, and the corresponding results can be easily extended to accommodate heterogeneous power constraints and higher modulation schemes.

Remark: The above system model is suitable for a wide range of practical WSNs, including clockdriven, event-driven, and inquiry-driven systems. For a clock-driven WSN, the remote node $o$ periodically (e.g., with period $T$ ) broadcasts beacons to activate simultaneous data transmissions from all nodes in $\mathbf{S}$. In this case, $N=|\mathbf{S}|$ and $T_{i}^{\text {limit }}=T$. For an event-driven WSN, a subset of $\mathbf{S}$ is activated simultaneously by the occurrence of an event. The activated nodes begin to transmit their sensed data roughly at the same time. Depending on the type of sensed data, e.g., voice, video, etc., there may be different deadlines for the transmissions from different sensors. Such deadlines are captured by $T_{i}^{\text {limit }}, i=1, \ldots, N$. For an inquiry-driven WSN, node $o$ broadcasts the inquiry request to the set $\mathbf{S}$, leading to a response from those sensors that have the desired answers. For a real-time inquiry, the desired information is usually needed by a certain time limit $T^{\text {limit }}$.

\section{$2.2 \quad$ Energy Consumption Model}

Consider the $i$ th sensor node with $B_{i}$ backlogged bits. The energy consumption at this node consists of transmission energy consumption and circuit energy consumption, i.e.,

$$
E_{i}=\left(P_{t i}+P_{c i}\right) T_{i}
$$

where $P_{c i}$ is the power consumed by the circuit at sensor $i$. Following a similar model to the one in [11], $P_{c i}$ can be written as

$$
P_{c i}=\alpha_{i}+\left(\frac{1}{\eta}-1\right) P_{t i}
$$

where $\alpha_{i}$ is a transmit-power-independent component that accounts for the power consumed by the digital-to-analog converter, the signal filters, and the modulator. $P_{P A i} \stackrel{\text { def }}{=}\left(\frac{1}{\eta}-1\right) P_{t i}$ is the power consumed by the power amplifier (PA), whose value is related to the transmission power via the efficiency of the PA $\eta$, where $\eta=\frac{P_{t i}}{P_{t i}+P_{P A i}}$. Physically, $\eta$ is determined by the drain efficiency of the RF power amplifier and the modulation scheme [11][20]. Substituting (2) into (1), the energy consumption of sensor $i$ is given by

$$
\begin{aligned}
E_{i} & =\frac{1}{\eta} P_{t i} T_{i}+\alpha_{i} T_{i} \\
& =\frac{1}{\eta}\left(P_{t i}+\alpha_{c i r i}\right) T_{i}
\end{aligned}
$$

where $\alpha_{c i r i}=\eta \alpha_{i}$ is defined as the equivalent circuit power consumption. For $N$ active sensor nodes, the total energy consumption is

$$
E_{\text {total }}=\sum_{i=1}^{N} E_{i}=\frac{1}{\eta} \sum_{i=1}^{N}\left(P_{t i}+\alpha_{c i r i}\right) T_{i} .
$$




\section{Problem Formulation and Numerical Solution}

The primary objective of our work is to find the optimal transmission power $P_{t i}^{o}$ and transmission time $T_{i}^{o}$ for each sensor node $i$ such that the total energy consumption for transmitting $\sum_{i=1}^{N} B_{i}$ bits is minimized while the QoS requirement of each transmission is satisfied. Formally, this is expressed as

$$
\left\{\begin{array}{l}
\min _{\left\{\mathbf{P}_{t}, \mathbf{T}\right\}} \sum_{i=1}^{N}\left(P_{t i}+\alpha_{c i r i}\right) T_{i} \\
\text { s.t. } \\
\left(\frac{E_{b}}{I_{0}}\right)_{i} \geq \gamma_{i}, \quad i=1, \ldots, N \\
0 \leq T_{i} \leq T_{i}^{\text {limit }}, \quad i=1, \ldots, N \\
0 \leq P_{t i} \leq P_{\max } . \quad i=1, \ldots, N
\end{array}\right.
$$

where $\mathbf{P}_{t} \stackrel{\text { def }}{=}\left(P_{t 1}, \ldots, P_{t N}\right)$ is the transmit power vector, $\mathbf{T} \stackrel{\text { def }}{=}\left(T_{1}, \ldots, T_{N}\right)$ is the transmission time vector, and $\left(\frac{E_{b}}{I_{0}}\right)_{i}$ is the received bit-energy-to-interference-density ratio at node $o$ for sensor $i$. This $\left(\frac{E_{b}}{I_{0}}\right)_{i}$ is given by

$$
\begin{aligned}
\left(\frac{E_{b}}{I_{0}}\right)_{i} & =\frac{W}{R_{i}} \frac{h_{i} P_{t i}}{\delta \sum_{j=1, j \neq i}^{N} h_{j} P_{t j}+N_{0} W} \\
& =\frac{W}{B_{i}} \frac{h_{i} P_{t i} T_{i}}{\delta \sum_{j=1, j \neq i}^{N} h_{j} P_{t j}+N_{0} W}
\end{aligned}
$$

where $R_{i}=\frac{B_{i}}{T_{i}}$ is the transmission rate under the assumption of BPSK modulation and $\delta$ is the orthogonality factor, representing multiple access interference (MAI) from the imperfect-orthogonal spreading codes and the asynchronous chips across simultaneous transmitting nodes. Typical values for $\delta$ are $\frac{2}{3}$ and 1 for a chip of rectangular or sinoide shape, respectively [15][16]. The second and third constraints in (5) come from the delay and transmit power upper bounds.

Because of the cross-product of $\mathbf{P}_{t}$ and $\mathbf{T}$ in the objective function and in the $\left(\frac{E_{b}}{I_{0}}\right)_{i}$ constraint, (5) is not a convex optimization problem. Hence, there is no guarantee that a locally optimal solution will indeed be globally optimal. We proceed to show that (5) can be put in a more standard form that reveals its special structure, for which an efficient numerical algorithm (geometric programming) is available. Moreover, as we show later, an approximate closed-form analytical solution is also possible due to the fact that the optimization problem can be solved sequentially, first with respect to power and then with respect to time.

Proposition 1: The problem formulation in (5) is a geometric programming, which can be transformed into a convex optimization problem of the so-called log-sum-exponential form so that the globally optimal solution can be efficiently derived by any numerical algorithm for convex optimization.

Proof: After some simple algebraic manipulations, (5) can be expressed as

$$
\left\{\begin{array}{l}
\min _{\left\{\mathbf{P}_{t}, \mathbf{T}\right\}} \sum_{i=1}^{N}\left(P_{t i}+\alpha_{c i r i}\right) T_{i} \\
\text { s.t. } \\
\delta B_{i} \gamma_{i}\left(W h_{i} P_{t i} T_{i}\right)^{-1} \sum_{j=1, j \neq i}^{N} h_{j} P_{t j}+B_{i} \gamma_{i}\left(W h_{i} P_{t i} T_{i}\right)^{-1} \leq 1, \quad i=1, \ldots, N \\
\frac{T_{i}}{T_{i}^{l i m i t}} \leq 1 \\
\frac{P_{t i}}{P_{\max }} \leq 1 \\
T_{i} \geq 0 \\
P_{t i} \geq 0
\end{array}\right.
$$


The objective function and all of the left-hand sides of the constraints in (8) are sums of monomials in $\left(\mathbf{P}_{t}, \mathbf{T}\right)$ with non-negative coefficients. These are known as posynomials ${ }^{1}$, and (8) can be solved using geometric programming [21]. The above form is still not a convex optimization problem. However, with a transformation of variables, (8) can be converted into an equivalent convex optimization problem. Let $x_{i}=\ln P_{t i}$ and $y_{i}=\ln T_{i}$. Taking the logarithms of both the objective function and constraints, (8) is transformed into the following equivalent problem:

$$
\left\{\begin{array}{l}
\min _{\{\mathbf{x}, \mathbf{y}\}} \log \sum_{i=1}^{N}\left[\exp \left(x_{i}+y_{i}\right)+\exp \left(\ln \alpha_{c i r i}+y_{i}\right)\right] \\
\text { s.t. } \\
\log \left[\sum_{j=1, j \neq i}^{N} \exp \left(x_{j}-x_{i}-y_{i}+\ln \delta B_{i} \gamma_{i} W^{-1} h_{i}^{-1} h_{j}\right)+\exp \left(\ln \left(B_{i} \gamma_{i} W^{-1} h_{i}^{-1}\right)-x_{i}-y_{i}\right)\right] \leq 0 \\
\log \exp \left(y_{i}-\ln T_{i}^{\text {limit }}\right) \leq 0, \\
\log \exp \left(x_{i}-\ln P_{\max }\right) \leq 0, \quad i=1, \ldots, N .
\end{array}\right.
$$

The log-sum-exponential function $f(\mathbf{z})=\log \left(\sum_{i=1}^{n} e^{z_{i}}\right)$, where $\mathbf{z}=\left(z_{1}, \ldots, z_{n}\right) \in \mathbf{R}^{n}$, is a convex function [21]. This implies that the affine mapping $g(\mathbf{s})=f(\mathbf{A} \mathbf{s}+\mathbf{B})$ preserves the convexity of $f(\mathbf{z})$. Hence, the objective function and all the constraints presented in (9) are convex, and so (9) is a convex optimization problem whose locally optimal solution $\left(\mathbf{x}^{o}, \mathbf{y}^{o}\right)$ is also globally optimal. Taking advantage of this useful property, efficient numerical algorithms for convex optimization problem, such as the primal-dual interior point method [21], can be used to solve for $\left(\mathbf{x}^{o}, \mathbf{y}^{o}\right)$. The globally optimal solution of (5) is simply given by $P_{t i}^{o}=\exp \left(x_{i}^{o}\right)$ and $T_{i}^{o}=\exp \left(y_{i}^{o}\right)$, for $i=1, \ldots, N$. Thus, Proposition 1 follows.

Note that the transformation from the posynomial-form geometric program (8) to the convexform problem (9) does not involve any computation; and the parameters for the two problems are the same. Therefore, the computational complexity is not increased by taking this transformation; it simply changes the form of the objective and constraint functions.

\section{Closed-Form Analytical Results}

The transformation of the optimization problem in (5) into (9) facilitates an accurate and very efficient numerical solution for finding the globally optimal transmission power and time for all active nodes in the system. In this section, we derive a closed-form analytical solution that may be viewed, in general, as a tight approximation of the exact solution. For all practical purposes, this analytical solution is indistinguishable from the numerical solution. The closed form of this solution makes it quite attractive for any real time transmit control operation.

The analytical solution is obtained by transforming the joint optimization problem in transmission power and time into two sequential sub-problems. This is achieved by first obtaining the optimal transmission power as an explicit function of the transmission time $\mathbf{T}$, for all feasible transmission times. Then, the optimal value of $\mathbf{T}$ is derived. Mathematically, this decoupling is described and justified in the following section.

\subsection{Mathematical Justification of the Decoupling Approach}

Let $\left(\mathbf{x}^{o}, \mathbf{y}^{o}\right)$ be the optimal solution to the minimization of a function $f(\mathbf{x}, \mathbf{y})$ over the feasible set $\Omega$, i.e., $f\left(\mathbf{x}^{o}, \mathbf{y}^{o}\right) \leq f(\mathbf{x}, \mathbf{y})$ for $\forall(\mathbf{x}, \mathbf{y}) \in \Omega$, where $\mathbf{x} \in \mathbf{R}^{n}, \mathbf{y} \in \mathbf{R}^{m}$, and $f: \mathbf{R}^{n} \times \mathbf{R}^{m} \rightarrow \mathbf{R}$. Let $\Omega_{1}$

\footnotetext{
${ }^{1} \mathrm{~A}$ posynomial in the variable $\mathbf{x}=\left(x_{1}, \ldots, x_{n}\right) \in \mathbf{R}^{n}$ is a linear combination of monomials with nonnegative coefficients. Formally, it is defined as $f(\mathbf{x})=\sum_{k=1}^{K} c_{k} x_{1}^{a_{k 1}} x_{2}^{a_{k 2}} \ldots x_{n}^{a_{k n}}$, where $c_{k} \geq 0$ and $a_{k j} \in \mathbf{R}, j=1,2, \ldots, n$.
} 
be the set composed of all feasible $\mathbf{x}$ in $\Omega$, i.e., $\Omega_{1}=\{\mathbf{x} \mid(\mathbf{x}, \mathbf{y}) \in \Omega\}$. For a given $\mathbf{x}_{0} \in \Omega_{1}$, define the function $F_{\mathbf{x}_{0}}(\mathbf{y})=f\left(\mathbf{x}_{0}, \mathbf{y}\right)$ and let the corresponding feasible set be $\Omega_{\mathbf{x}_{0}}=\left\{\mathbf{y} \mid\left(\mathbf{x}_{0}, \mathbf{y}\right) \in \Omega\right\}$. Assuming the minimum value of $F_{\mathbf{x}_{0}}(\mathbf{y})$ exists over $\Omega_{\mathbf{x}_{0}}$, then there must be some $\mathbf{y}_{0}^{*} \in \Omega_{\mathbf{x}_{0}}$ for which $F_{\mathbf{x}_{0}}\left(\mathbf{y}_{0}^{*}\right) \leq F_{\mathbf{x}_{0}}(\mathbf{y})$ for $\forall \mathbf{y} \in \Omega_{\mathbf{x}_{0}}$. Define the multi-dimensional function $\mathbf{g}(\mathbf{x})$ as the mapping from $\mathbf{x}_{0}$ to $\mathbf{y}_{0}^{*}$, i.e., for any $\mathbf{x} \in \Omega_{1}$, the corresponding optimal solution to the problem $\min F_{\mathbf{x}}(\mathbf{y})$ over the feasible set $\Omega_{\mathbf{x}}$ is given by $\mathbf{y}^{*}=\mathbf{g}(\mathbf{x})=\left(g_{1}(\mathbf{x}), \ldots, g_{m}(\mathbf{x})\right)$, so that $F_{\mathbf{x}}(\mathbf{g}(\mathbf{x})) \leq F_{\mathbf{x}}(\mathbf{y})$ for $\forall \mathbf{y} \in \Omega_{\mathbf{x}}$. Regarding the optimal solution to $\min f(\mathbf{x}, \mathbf{y})$ over $\Omega$, we have the following proposition:

Proposition 2: Define $h(\mathbf{x})=f(\mathbf{x}, \mathbf{g}(\mathbf{x}))$. Let $\mathbf{x}^{*}$ be the (globally) optimal solution to problem

$$
\min h(\mathbf{x}) \text { over the feasible set } \Omega_{1} \text {, }
$$

i.e., $h\left(\mathbf{x}^{*}\right) \leq h(\mathbf{x}), \forall \mathbf{x} \in \Omega_{1}$. Then, $f\left(\mathbf{x}^{*}, \mathbf{g}\left(\mathbf{x}^{*}\right)\right)=f\left(\mathbf{x}^{o}, \mathbf{y}^{o}\right)$, i.e., $\left(\mathbf{x}^{*}, \mathbf{g}\left(\mathbf{x}^{*}\right)\right)$ is also the globally optimal solution to the problem

$$
\min f(\mathbf{x}, \mathbf{y}) \text { over } \Omega \text {. }
$$

Proof: Because $\mathbf{x}^{*} \in \Omega_{1}$ and $\mathbf{g}\left(\mathbf{x}^{*}\right) \in \Omega_{\mathbf{x}^{*}}$, it is clear that $\left(\mathbf{x}^{*}, \mathbf{g}\left(\mathbf{x}^{*}\right)\right) \in \Omega$. Because $\left(\mathbf{x}^{o}, \mathbf{y}^{o}\right)$ is the optimal solution to (11), it immediately follows that $f\left(\mathbf{x}^{o}, \mathbf{y}^{o}\right) \leq f\left(\mathbf{x}^{*}, \mathbf{g}\left(\mathbf{x}^{*}\right)\right)$. On the other hand, because $\mathbf{x}^{*}$ is the optimal solution to (10), it follows that for $\forall \mathbf{x} \in \Omega_{1}$,

$$
h\left(\mathbf{x}^{*}\right)=f\left(\mathbf{x}^{*}, \mathbf{g}\left(\mathbf{x}^{*}\right)\right) \leq h(\mathbf{x})=f(\mathbf{x}, \mathbf{g}(\mathbf{x})) .
$$

Therefore, it follows that

$$
f\left(\mathbf{x}^{*}, \mathbf{g}\left(\mathbf{x}^{*}\right)\right) \leq f\left(\mathbf{x}^{o}, \mathbf{g}\left(\mathbf{x}^{o}\right)\right)=F_{\mathbf{x}^{o}}\left(\mathbf{g}\left(\mathbf{x}^{o}\right)\right)
$$

For any given $\mathbf{x}, \mathbf{y}^{*}=\mathbf{g}(\mathbf{x})$ provides the optimal (minimum) value of $F_{\mathbf{x}}(\mathbf{y})$ over the feasible set $\Omega_{\mathbf{x}}$. Therefore,

$$
F_{\mathbf{x}^{o}}\left(\mathbf{g}\left(\mathbf{x}^{o}\right)\right) \leq F_{\mathbf{x}^{o}}(\mathbf{y}), \quad \forall \mathbf{y} \in \Omega_{\mathbf{x}^{o}} .
$$

Because $\left(\mathbf{x}^{o}, \mathbf{y}^{o}\right) \in \Omega$, there must be $\mathbf{y}^{o} \in \Omega_{\mathbf{x}^{o}}$. Therefore,

$$
F_{\mathbf{x}^{o}}\left(\mathbf{g}\left(\mathbf{x}^{o}\right)\right) \leq F_{\mathbf{x}^{o}}\left(\mathbf{y}^{o}\right)=f\left(\mathbf{x}^{o}, \mathbf{y}^{o}\right)
$$

Combining (15) and (13), it follows that

$$
f\left(\mathbf{x}^{*}, \mathbf{g}\left(\mathbf{x}^{*}\right)\right) \leq f\left(\mathbf{x}^{o}, \mathbf{y}^{o}\right) .
$$

But $f\left(\mathbf{x}^{o}, \mathbf{y}^{o}\right) \leq f\left(\mathbf{x}^{*}, \mathbf{g}\left(\mathbf{x}^{*}\right)\right)$. Thus, it must be that $f\left(\mathbf{x}^{*}, \mathbf{g}\left(\mathbf{x}^{*}\right)\right)=f\left(\mathbf{x}^{o}, \mathbf{y}^{o}\right)$.

Analytically, Proposition 2 suggests that the optimization of the two-variable problem (11) can be divided into two sequential sub-problems. In the first sub-problem, $\mathbf{x}$ is treated as a given parameter and $\mathbf{y}$ as the optimization variable. For a given $\mathbf{x}$, the optimal $\mathbf{y}$ that minimizes the objective function is presented as a function of $\mathbf{x}$, i.e., $\mathbf{g}(\mathbf{x})$. The optimization results of sub-problem 1 is then forwarded to the configuration of sub-problem 2 , where the objective function is transformed to $f(\mathbf{x}, \mathbf{g}(\mathbf{x}))$ and the optimization is conducted over $\mathbf{x}$. Proposition 2 proves that the optimal solution of the original problem is obtained from the results of sub-problems 1-2 as $\left(\mathbf{x}^{*}, \mathbf{g}\left(\mathbf{x}^{*}\right)\right)$. Taking advantage of this property, the analysis of (5) proceeds as follows. 


\subsection{Sub-Problem 1: Parametric Solution for Optimal Transmission Power}

Treating the transmission time vector $\mathbf{T}$ as a given system parameter with $T_{i} \leq T_{i}^{\text {limit }}$, problem (5) is equivalent to the following linear programming problem:

$$
\left\{\begin{array}{l}
\min _{\left\{P_{t 1}, \ldots, P_{t N}\right\}} \sum_{i=1}^{N} P_{t i} T_{i} \\
\text { s.t. } \\
\left(1+\frac{\delta B_{i} \gamma_{i}}{W T_{i}}\right) h_{i} P_{t i}-\frac{\delta B_{i} \gamma_{i}}{W T_{i}} \sum_{j=1}^{N} h_{j} P_{t j} \geq \frac{B_{i} \gamma_{i} N_{0}}{T_{i}}, \quad i=1, \ldots, N \\
P_{t i} \leq P_{\max }
\end{array}\right.
$$

Regarding the optimal solution to (17), we have the following proposition.

Proposition 3: If the optimal solution to (17) exists, i.e., the feasible set depicted by the constraints in (17) is not empty, then this optimal solution is the solution to the following set of linear equations

$$
\left(1+\frac{\delta B_{i} \gamma_{i}}{W T_{i}}\right) h_{i} P_{t i}-\frac{\delta B_{i} \gamma_{i}}{W T_{i}} \sum_{j=1}^{N} h_{j} P_{t j}=\frac{B_{i} \gamma_{i} N_{0} W}{W T_{i}}, \quad i=1, \ldots, N
$$

Proof: Let $f_{i}\left(\mathbf{P}_{t}\right) \stackrel{\text { def }}{=}\left(1+\frac{\delta B_{i} \gamma_{i}}{W T_{i}}\right) h_{i} P_{t i}-\frac{\delta B_{i} \gamma_{i}}{W T_{i}} \sum_{j=1}^{N} h_{j} P_{t j}, i=1, \ldots, N$. Its first-order partial derivations are

$$
\frac{\partial f_{i}}{\partial P_{t i}}=h_{i}>0
$$

and

$$
\frac{\partial f_{i}}{\partial P_{t j}}=-\frac{B_{i} \gamma_{i}}{W T_{i}} h_{j}<0, \quad \text { for } j \neq i
$$

The derivations indicate that $f_{i}\left(\mathbf{P}_{t}\right)$ is a strict mono-increasing function of $P_{t i}$ and a strict monodecreasing function of $P_{t j}, j \neq i$.

Let the optimal solution to $(17)$ be $\mathbf{P}_{t}^{o}=\left(P_{t 1}^{o}, \ldots, P_{t N}^{o}\right)$. Then it follows that $\sum_{i=1}^{N} P_{t i}^{o} T_{i} \leq$ $\sum_{i=1}^{N} P_{t i} T_{i}$ for any feasible transmit power vector $\mathbf{P}_{t}=\left(P_{t 1}, \ldots, P_{t N}\right)$. Suppose for some node $k, 1 \leq$ $k \leq N, f_{k}\left(\mathbf{P}_{t}^{o}\right)>\frac{B_{k} \gamma_{k} N_{0}}{T_{k}}$. Then, for this node, there must be some increment $\triangle P_{t k}>0$ such that replacing $P_{t k}^{o}$ by $P_{t k}^{o^{\prime}}=P_{t k}^{o}-\triangle P_{t k}$ while keeping the transmit power of other nodes intact results in $f_{k}\left(\mathbf{P}_{t}^{o^{\prime}}\right) \geq \frac{B_{k} \gamma_{k} N_{0}}{T_{k}}$ and $f_{i}\left(\mathbf{P}_{t}^{o^{\prime}}\right) \geq \frac{B_{i} \gamma_{i} N_{0}}{T_{i}}$, for $i \neq k$, where $\mathbf{P}_{t}^{o^{\prime}}=\left(P_{t 1}^{o}, \ldots, P_{t k}^{o}-\triangle P_{t k}, \ldots, P_{t N}^{o}\right)$. Therefore, $\mathbf{P}_{t}^{o^{\prime}}$ is also a feasible solution to problem (17). However, it is easy to show that $\sum_{i=1}^{N} P_{t i}^{o^{\prime}} T_{i}$ is strictly smaller than $\sum_{i=1}^{N} P_{t i}^{o} T_{i}$ by $\triangle P_{t k} T_{k}$, leading to a conflict with the supposition that $\mathbf{P}_{t}^{o}$ is the optimal solution that minimizes the objective function $\sum_{i=1}^{N} P_{t i} T_{i}$. Therefore, there can not be any node $k$ that does not meet the equality in the first constraint in (17). Then Proposition 3 follows.

After some mathematical manipulations of (18), we arrive at

$$
h_{i} P_{t i}=\frac{\delta B_{i} \gamma_{i}}{W T_{i}+\delta B_{i} \gamma_{i}} \sum_{j=1}^{N} h_{j} P_{t j}+\frac{B_{i} \gamma_{i}}{W T_{i}+\delta B_{i} \gamma_{i}} N_{0} W, \quad i=1, \ldots, N
$$

Define the power index of node $i$ as:

$$
g_{i} \stackrel{\text { def }}{=} \frac{\delta B_{i} \gamma_{i}}{W T_{i}+\delta B_{i} \gamma_{i}}
$$

Equation (21) can be rewritten as

$$
h_{i} P_{t i}=g_{i} \sum_{j=1}^{N} h_{j} P_{t j}+\frac{1}{\delta} g_{i} N_{0} W, \quad i=1, \ldots, N .
$$


Summing over $i$ leads to

$$
\sum_{i=1}^{N} h_{i} P_{t i}=g_{\Sigma} \sum_{j=1}^{N} h_{j} P_{t j}+\frac{1}{\delta} g_{\Sigma} N_{0} W,
$$

where $g_{\Sigma} \stackrel{\text { def }}{=} \sum_{i=1}^{N} g_{i}$. Therefore, the solution to (18), and also the optimal solution to problem (17) if it exists, is simply given by

$$
P_{t i}=\frac{\delta^{-1} h_{i}^{-1} g_{i}}{1-g_{\Sigma}} N_{0} W
$$

Assuming $N_{0} W=1$, i.e., normalizing $P_{t i}$ by the background AWGN, (25) is further simplified to

$$
P_{t i}=\frac{\delta^{-1} h_{i}^{-1} g_{i}}{1-g_{\Sigma}}, \quad i=1, \ldots, N
$$

Given any feasible transmission time vector $\mathbf{T},(26)$ presents the optimal transmit power vector in terms of $\mathbf{T}$ if such optimal solution exists. Regarding the second constraint in (17), a necessary condition for the existence of the optimal solution is given by

$$
P_{t i}=\frac{\delta^{-1} h_{i}^{-1} g_{i}}{1-g_{\Sigma}} \leq P_{\max }
$$

which leads to

$$
g_{i} \leq \delta\left(1-g_{\Sigma}\right) h_{i} P_{\max }, \quad i=1, \ldots, N .
$$

The inequality (28) depicts a polyhedron in $\mathbf{R}_{+}^{N}$ within which a feasible solution to (17) exists (thus, the optimal solution exists). Summing over $i$ in (28), we have

$$
g_{\Sigma} \leq \frac{\delta P_{\max } h_{\Sigma}}{1+\delta P_{\max } h_{\Sigma}}<1
$$

where $h_{\Sigma} \stackrel{\text { def }}{=} \sum_{i=1}^{N} h_{i}$. To provide a tractable closed-form solution, we relax (28) into

$$
g_{i} \leq \delta h_{i} P_{\max }, \quad i=1, \ldots, N
$$

Note that this relaxation may result in transmission powers for some sensor nodes that exceed the upper bound $P_{\max }$ if the received signal quality constraints are to be satisfied for all nodes. However, for a typical CDMA-based WSN application, which is characterized by low data transmission rates, large spread spectrum bandwidth, and a relatively small SINR requirement, the $g_{i}$ 's are very small and $g_{\Sigma} \ll 1$. Consequently, the expansion of the feasible set through (30) will result in a tight approximation to the original polyhedron in (28), as will be demonstrated later in Section 6 .

To summarize the results of this section, for any given feasible transmission time $\mathbf{T}$, the parametric optimal transmit power is given by (26). In order to guarantee the existence of this optimal power allocation, (30) and (29) must be satisfied, where $g_{i}$ is defined in (22) and $g_{\Sigma}=\sum_{i=1}^{N} g_{i}$.

\subsection{Sub-Problem 2: Optimization of Transmission Time}

¿From (22), it is clear that for given $B_{i}, \gamma_{i}, W$, and $\delta$, the power index $g_{i}$ and the transmission time $T_{i}$ are equivalent measures in the sense that there is a one-to-one mapping between $g_{i}$ and $T_{i}$ :

$$
T_{i}=\frac{\delta B_{i} \gamma_{i}}{W g_{i}}\left(1-g_{i}\right)
$$


In the following optimization, it is more mathematically convenient to work with $g_{i}$. Let $\mathbf{g} \stackrel{\text { def }}{=}$ $\left(g_{1}, \ldots, g_{N}\right)$. The problem of determining the optimal value of $\mathbf{g}$ is formulated by substituting (31), (26), and the constraints (30) and (29) into the original optimization problem (5). This results in

$$
\left\{\begin{array}{l}
\min _{\left\{g_{1}, \ldots, g_{N}\right\}}\left\{h\left(g_{1}, \ldots, g_{N}\right) \stackrel{\text { def }}{=} \sum_{i=1}^{N}\left(\frac{\delta^{-1} h_{i}^{-1} g_{i}}{1-g_{\Sigma}}+\alpha_{c i r i}\right) \frac{\delta B_{i} \gamma_{i}}{W g_{i}}\left(1-g_{i}\right)\right\} \\
\text { s.t. } \\
\frac{\delta B_{i} \gamma_{i}}{\delta B_{i} \gamma_{i}+W T_{i}^{l i m i t}} \leq g_{i} \leq \delta h_{i} P_{\max }, \quad i=1, \ldots, N \\
\sum_{i=1}^{N} g_{i} \leq \frac{\delta P_{\max } h_{\Sigma}}{1+\delta P_{\max } h_{\Sigma}}
\end{array}\right.
$$

where the lower bound on $g_{i}$ in the first constraint comes from the delay bound requirement $T_{i}$. In most cases, (32) is a well-formulated problem, meaning that the upper bound requirement on $g_{i}$ is larger than its lower bound, so the feasible solution set to (32) is not empty. However, in the case when both $h_{i}$ and $P_{\max }$ are extremely small to the extent that the upper bound on $g_{i}$ is smaller than its lower bound, the feasible set to (32) is null, and no solution exists to problem (5).

Rewriting the objective function $h\left(g_{1}, \ldots, g_{N}\right)$ in $(32)$ by expanding the products results in

$$
h\left(g_{1}, \ldots, g_{N}\right)=\sum_{i=1}^{N} \frac{h_{i}^{-1} B_{i} \gamma_{i}\left(1-g_{i}\right)}{\left(1-g_{\Sigma}\right) W}+\sum_{i=1}^{N} \frac{\alpha_{c i r i} \delta B_{i} \gamma_{i}}{W g_{i}}-\sum_{i=1}^{N} \frac{\alpha_{c i r i} \delta B_{i} \gamma_{i}}{W}
$$

As stated in the formulation of sub-problem 1, for a typical WSN application, $g_{i} \ll 1$. Therefore, (33) is tightly approximated by

$$
\begin{aligned}
h\left(g_{1}, \ldots, g_{N}\right) & \approx \frac{\sum_{i=1}^{N} h_{i}^{-1} B_{i} \gamma_{i}}{\left(1-g_{\Sigma}\right) W}+\sum_{i=1}^{N} \frac{\alpha_{c i r i} \delta B_{i} \gamma_{i}}{W g_{i}}-\sum_{i=1}^{N} \frac{\alpha_{i} \delta B_{i} \gamma_{i}}{W} \\
& =\frac{K}{1-g_{\Sigma}}+\sum_{i=1}^{N} \frac{\alpha_{c i r i} A_{i}}{g_{i}}-\sum_{i=1}^{N} \alpha_{c i r i} A_{i}
\end{aligned}
$$

where $A_{i} \stackrel{\text { def }}{=} \frac{\delta B_{i} \gamma_{i}}{W}$ is a node-dependent constant and $K \stackrel{\text { def }}{=} \sum_{i=1}^{N} \delta^{-1} h_{i}^{-1} A_{i}$ is a system-dependent constant.

Proposition 4: The function $h\left(g_{1}, \ldots, g_{N}\right)$ in (34) is strictly convex.

Proof: The first-order partial derivative of $h\left(g_{1}, \ldots, g_{N}\right)$ with respect to $g_{i}, i=1, \ldots, N$, is given by

$$
\frac{\partial h}{\partial g_{i}}=\frac{K}{\left(1-g_{\Sigma}\right)^{2}}-\frac{\alpha_{c i r i} A_{i}}{g_{i}^{2}} .
$$

The second-order partial deviation is given by

$$
\frac{\partial^{2} h}{\partial g_{i}^{2}}=\frac{2 K}{\left(1-g_{\Sigma}\right)^{3}}+\frac{2 \alpha_{c i r i} A_{i}}{g_{i}^{3}}
$$

and for $i \neq j$

$$
\frac{\partial^{2} h}{\partial g_{i} \partial g_{j}}=\frac{2 K}{\left(1-g_{\Sigma}\right)^{3}}
$$

Therefore, the Heissian of $h\left(g_{1}, \ldots, g_{N}\right)$ is given by ${ }^{2}$

$$
\nabla^{2} h\left(g_{1}, \ldots, g_{N}\right)=\frac{2 K}{\left(1-g_{\Sigma}\right)^{3}} \mathbf{I}+\mathbf{D}
$$

\footnotetext{
${ }^{2}$ The element $a_{i j}$ of the Heissian of a multi-variable function $f\left(x_{1}, \ldots, x_{n}\right)$ is defined as $a_{i j}=\frac{\partial^{2} f}{\partial x_{i} \partial x_{j}}$, for $i, j=$ $1, \ldots, n$.
} 
where $\mathbf{I}$ is an $N \times N$ matrix with all elements equal to 1 and $\mathbf{D}$ is an $N \times N$ diagonal matrix whose $i$ th diagonal element is $\frac{2 \alpha_{c i r i}}{g_{i}^{3}}$. For any non-zero vector $\mathbf{v}=\left(v_{1}, \ldots, v_{N}\right) \in \mathbf{R}^{N}$, it is easy to show that

$$
\begin{aligned}
\mathbf{v} \cdot \mathbf{I} \cdot \mathbf{v}^{T} & =\sum_{i=1}^{N} \sum_{j=1}^{N} v_{i} v_{j} \\
& =\left(v_{1}+\ldots+v_{N}\right)^{2} \geq 0
\end{aligned}
$$

and

$$
\mathbf{v} \cdot \mathbf{D} \cdot \mathbf{v}^{T}=\sum_{i=1}^{N} \frac{2 \alpha_{c i r i} A_{i}}{g_{i}^{3}} v_{i}^{2}>0
$$

Therefore, $\nabla^{2} h\left(g_{1}, \ldots, g_{N}\right)$ is positive definite, and thus $h\left(g_{1}, \ldots, g_{N}\right)$ is a strictly convex function of $\left(g_{1}, \ldots, g_{N}\right)$.

Replacing $h\left(g_{1}, \ldots, g_{N}\right)$ in the objective function in (32) by its approximation in (34), we arrive at the following convex optimization problem

$$
\left\{\begin{array}{l}
\min _{\left\{g_{1}, \ldots, g_{N}\right\}} \frac{K}{1-g_{\Sigma}}+\sum_{i=1}^{N} \frac{\alpha_{c i r i} A_{i}}{g_{i}}-\sum_{i=1}^{N} \alpha_{c i r i} A_{i} \\
\text { s.t. } \\
\frac{\delta B_{i} \gamma_{i}}{\delta B_{i} \gamma_{i}+W T_{i}^{l i m i t}} \leq g_{i} \leq \delta h_{i} P_{\max }, \quad i=1, \ldots, N \\
\sum_{i=1}^{N} g_{i} \leq \frac{\delta P_{\max } h_{\Sigma}}{1+\delta P_{\max } h_{\Sigma}} .
\end{array}\right.
$$

Since (34) is a tight approximation, we can also expect that the optimal solution to (41) will be a tight approximation to the optimal solution of (32).

The optimal solution $\left(g_{1}^{o}, \ldots, g_{N}^{o}\right)$ to the constrained problem $(41)$ is related to the solution of the unconstrained minimization of $h(\mathbf{g})$. Being strictly convex, $h\left(g_{1}, \ldots, g_{N}\right)$ must have only one unconstrained minimum solution, which can be derived by solving the following equation set:

$$
\frac{\partial h}{\partial g_{i}}=\frac{K}{\left(1-g_{\Sigma}\right)^{2}}-\frac{\alpha_{c i r i} A_{i}}{g_{i}^{2}}=0, \quad i=1, \ldots, N .
$$

Through some mathematical manipulations, it can be shown that the unconstrained optimum solution $\left(g_{u 1}^{o}, \ldots, g_{u N}^{o}\right)$ to $h\left(g_{1}, \ldots, g_{N}\right)$ is given by

$$
g_{u i}^{o}=\frac{\sqrt{\alpha_{c i r i} A_{i}}}{\sqrt{K}+\sum_{i=1}^{N} \sqrt{\alpha_{c i r i} A_{i}}}, \quad i=1, \ldots, N
$$

Because of the convexity of $h(\mathbf{g})$, if any of the $g_{u i}^{o}$ in (43) violates the upper or the lower bound on $g_{i}$ in (41), then the corresponding constrained optimal solution $g_{i}^{o}$ must itself be the upper or the lower bound, depending on which bound is being violated. Accordingly, the optimal solution to the constrained problem is given in the following proposition.

Proposition 5: Let $\left(g_{1}^{o}, \ldots, g_{N}^{o}\right)$ denote the optimal solution to (41). Let $g_{i}^{u p p} \stackrel{\text { def }}{=} \delta h_{i} P_{\max }$ and

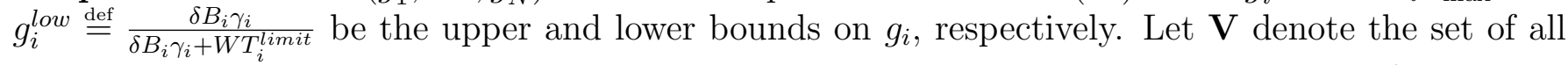
active nodes, and let $\mathbf{U}$ denote the set of active nodes for which $g_{i}^{o}=g_{i}^{\text {upp }}$ or $g_{i}^{o}=g_{i}^{\text {low }}$. Define $t_{1} \stackrel{\text { def }}{=} 1-\sum_{j \in \mathbf{U}} g_{j}^{o}$ and $t_{2} \stackrel{\text { def }}{=} \frac{\delta P_{\max } h_{\Sigma}}{1+\delta P_{\max } h_{\Sigma}}-\sum_{j \in \mathbf{U}} g_{j}^{o}$. Then for $i=1, \ldots, N$,

1. If $\sum_{i=1}^{N} g_{i}^{o}<\frac{\delta P_{\max } h_{\Sigma}}{1+\delta P_{\max } h_{\Sigma}}$, then $g_{i}^{o} \in\left\{g_{i}^{u p p}, \frac{t_{1} \sqrt{\alpha_{i} A_{i}}}{\sqrt{K}+\sum_{j \in \mathbf{V}-\mathbf{U}} \sqrt{\alpha_{j} A_{j}}}, g_{i}^{\text {low }}\right\}$.

2. If $\sum_{i=1}^{N} g_{i}^{o}=\frac{\delta P_{\max } h_{\Sigma}}{1+\delta P_{\max } h_{\Sigma}}$, then $g_{i}^{o} \in\left\{g_{i}^{u p p}, \frac{t_{2} \sqrt{\alpha_{i} A_{i}}}{\sum_{j \in \mathbf{V}-\mathbf{U}} \sqrt{\alpha_{j} A_{j}}}, g_{i}^{\text {low }}\right\}$. 
Note: In either of these two cases, at least one $g_{i}^{o}$ will equal the intermediate value. Proof: The proof actually provides a recursive algorithm for solving for $g_{i}^{o}$.

Case 1: First, we consider the case when $\sum_{i=1}^{N} g_{i}^{o}<\frac{\delta P_{\max } h_{\Sigma}}{1+\delta P_{\max } h_{\Sigma}}$. Let $\mathbf{U}$ be initially empty. Because of the strict convexity of $h(\mathbf{g})$, if for some $i$, the unconstrained optimal solution $g_{u i}^{o}$ exceeds its upper bound, i.e., $g_{u i}^{o}>g_{i}^{u p p}$, then the constrained optimal solution must be $g_{i}^{o}=g_{i}^{u p p}$. Similarly, if $g_{u i}^{o}<g_{i}^{\text {low }}$, then $g_{i}^{o}=g_{i}^{\text {low }}$. Such nodes, whose unconstrained optimal solutions exceed their upper or lower bounds are added to the set $\mathbf{U}$. With the knowledge of $g_{i}^{o}$ for $i \in \mathbf{U}$, the objective function in (41) is equivalent to the following function

$$
h^{\prime}(\mathbf{V}-\mathbf{U})=\frac{K}{t_{1}-g_{\Sigma}^{\prime}}+\sum_{i \in \mathbf{V}-\mathbf{U}} \frac{\alpha_{c i r i} A_{i}}{g_{i}}+\sum_{i \in \mathbf{U}} \frac{\alpha_{c i r i} A_{i}}{g_{i}^{o}}-\sum_{i=1}^{N} \alpha_{c i r i} A_{i},
$$

where $g_{\Sigma}^{\prime} \stackrel{\text { def }}{=} \sum_{i \in \mathbf{V}-\mathbf{U}} g_{i}$. Because $g_{i}^{o}$ is known for any $i \in \mathbf{U}$, replacing the objective function in (41) by (44) leads to an inherited problem that is of the same form as (41) except that the number of variables is reduced from $|\mathbf{V}|$ to $|\mathbf{V}-\mathbf{U}|$. With some mathematical manipulations, it can be shown that the unconstrained optimal solution to (44) is given by

$$
g_{u i}^{o^{\prime}}=\frac{t_{1} \sqrt{\alpha_{c i r i} A_{i}}}{\sqrt{K}+\sum_{j \in \mathbf{V}-\mathbf{U}} \sqrt{\alpha_{c i r j} A_{j}}}, \quad i \in \mathbf{V}-\mathbf{U}
$$

which is a recurrent version of (43) in terms of $t_{1}$ and $\mathbf{U}$. The above process is repeated and the values of $t_{1}$ and $\mathbf{U}$ are updated based on the newly computed values of $g_{i}^{o}$ until all remaining unconstrained solutions $g_{u i}^{o}, i \in \mathbf{V}-\mathbf{U}$, of the inherited problem meet their respective upper and lower bounds. In the last iteration, the remaining $g_{i}^{o}$ 's, $i \in \mathbf{V}-\mathbf{U}$, are equal to their unconstrained counterparts given by $(45)$.

Once all the $g_{i}^{o}$ have been computed, it should be verified that $\sum_{i=1}^{N} g_{i}^{o}<\frac{\delta P_{\max } h_{\Sigma}}{1+\delta P_{\max } h_{\Sigma}}$. If this is not the case, then the solution of $g_{i}^{o}$ falls into the next case.

Case 2: Consider the case when $\sum_{i=1}^{N} g_{i}^{o}=\frac{\delta P_{\max } h_{\Sigma}}{1+\delta P_{\max } h_{\Sigma}}$. In this case, the objective function in (41) degenerates into the following function

$$
h_{2}(\mathbf{g}) \stackrel{\text { def }}{=} K\left(1+\delta P_{\max } h_{\Sigma}\right)+\sum_{i=1}^{N} \frac{\alpha_{\text {ciri }} A_{i}}{g_{i}}-\sum_{i=1}^{N} \alpha_{c i r i} A_{i} .
$$

Accordingly, (41) is equivalent to the following problem

$$
\left\{\begin{array}{l}
\min _{\left\{g_{1}, \ldots, g_{N}\right\}} \sum_{i=1}^{N} \frac{\alpha_{c i r i} A_{i}}{g_{i}} \\
\text { s.t. } \\
\sum_{i=1}^{N} g_{i}=\frac{\delta P_{\max } h_{\Sigma}}{1+\delta P_{\max } h_{\Sigma}}, \\
\frac{\delta B_{i} \gamma_{i}}{\delta B_{i} \gamma_{i}+W T_{i}^{\text {limit }}} \leq g_{i} \leq \delta h_{i} P_{\max }, \quad i=1, \ldots, N .
\end{array}\right.
$$

In this case, it is easy to show that

$$
\nabla^{2} h_{2}\left(g_{1}, \ldots, g_{N}\right)=\operatorname{diag}\left(\frac{2 \alpha_{c i r 1} A_{1}}{g_{2}^{3}}, \ldots, \frac{2 \alpha_{c i r N} A_{N}}{g_{N}^{3}}\right),
$$

which is a positive definite matrix. Therefore, $h_{2}(\mathbf{g})$ is a strictly convex function. Under the condition $\sum_{i=1}^{N} g_{i}^{o}=\frac{\delta P_{\max } h_{\Sigma}}{1+\delta P_{\max } h_{\Sigma}}$, the optimal unbounded (i.e., ignoring the upper and lower bounds on $g_{i}$ ) solution to $h_{2}(\mathbf{g})$ is given by

$$
g_{u i}^{o}=\frac{\frac{\delta P_{\max } h_{\Sigma}}{1+\delta P_{\max } h_{\Sigma}} \sqrt{\alpha_{c i r i} A_{i}}}{\sum_{j=1}^{N} \sqrt{\alpha_{c i r j} A_{j}}}
$$


Accounting for the upper- and lower-bound constraints of $g_{i}$ and following a similar process to case 1 , it can be found that $g_{i}^{o}$ is equal to $g_{i}^{u p p}, g_{i}^{\text {low }}$, or

$$
g_{i}^{o}=\frac{t_{2} \sqrt{\alpha_{c i r i} A_{i}}}{\sum_{j \in \mathbf{V}-\mathbf{U}} \sqrt{\alpha_{c i r j} A_{j}}}, \quad i \in \mathbf{V}-\mathbf{U} .
$$

If in one of the computational cycles $g_{i}^{o}$ is found to be equal to $\delta h_{i} P_{\max }$ or $\frac{\delta B_{i} \gamma_{i}}{\delta B_{i} \gamma_{i}+W T_{\text {limit }}^{i}}$ for all $i=1, \ldots, N$, then there is no feasible solution to (41) because the constraint $\sum_{i=1}^{N} g_{i}^{o}=\frac{\delta P_{\max } h_{\Sigma}}{1+\delta P_{\max } h_{\Sigma}}$ can not be satisfied.

The above proof actually describes the "mechanics" for computing the optimal solution to (41). A pseudo-code representation of the computational algorithm is outlined in Table 1. The following example further illustrates the operation of this algorithm.

Example: Let $N=5, K=144, \alpha_{\text {cir } 1}=\ldots=\alpha_{\text {cir } 5}=1, A_{1}=A_{2}=A_{3}=1, A_{4}=4, A_{5}=9$. The upper bounds are set to $g_{i}^{\text {upp }}=0.1,0.1,0.055,0.05,0.1$ for $i=1, \ldots, 5$, respectively. Let $g_{i}^{\text {low }}=0.01$ for all nodes, and let $\frac{\delta P_{\max } h_{\Sigma}}{1+\delta P_{\max } h_{\Sigma}}=0.9$. To determine $g_{i}^{o}$ for $i=1, \ldots, 5$, we first assume that $\sum_{i=1}^{5} g_{i}^{o}<0.9$ and consider case 1 of Proposition 5 (once the $g_{i}^{o}$ 's have been computed, we can verify whether or not case 1 is the appropriate case). we initially set $\mathbf{U}=\emptyset$ and $t_{1}=1$.

In the first iteration, according to (45), we have $g_{u 1}^{o}=g_{u 2}^{o}=g_{u 3}^{o}=0.05, g_{u 4}^{o}=0.1$, and $g_{u 5}^{o}=0.15$. Comparing these values with their respective upper and lower bounds, we find that $g_{u 4}^{o}$ and $g_{u 5}^{o}$ violate their upper bounds. Therefore, we set $g_{4}^{o}=g_{4}^{u p p}=0.05$ and $g_{5}^{o}=g_{5}^{u p p}=0.1$ as their final values. Updating $\mathbf{U}$ and $t_{1}$, we have $\mathbf{U}=\{4,5\}$ and $t_{1}=0.85$.

In the second iteration, we have $g_{u 1}^{o}=g_{u 2}^{o}=g_{u 3}^{o}=0.05667$. Comparing these values with their respective upper and lower bounds, we notice that $g_{u 3}^{o}$ violates its upper bound. Therefore, $g_{3}^{o}=g_{3}^{u p p}=0.055$. Updating $\mathbf{U}$ and $t_{1}$, we have $\mathbf{U}=\{3,4,5\}$ and $t_{1}=0.795$.

Finally, in the third iteration, we have $g_{u 1}^{o}=g_{u 2}^{o}=0.0568$. Since both of these values are compliant with their upper and lower bounds, $g_{1}^{o}=g_{u 1}^{o}=0.0568$ and $g_{2}^{o}=g_{u 2}^{o}=0.0568$. After verifying that $\sum_{i=1}^{5} g_{i}^{o}<0.9$, the algorithm terminates.

Once the $g_{i}^{o}$ 's have been computed, Proposition 2 indicates that the optimal transmit power and transmission time are obtained by combining (26), (31), and Proposition 5:

$$
\begin{aligned}
P_{t i}^{o} & =\frac{\delta^{-1} h_{i}^{-1} g_{i}^{o}}{1-g_{\Sigma}^{o}} \\
T_{i}^{o} & =\frac{\delta B_{i} \gamma_{i}}{W g_{i}^{o}}\left(1-g_{i}^{o}\right), \quad i=1, \ldots, N
\end{aligned}
$$

where $g_{\Sigma}^{o} \stackrel{\text { def }}{=} \sum_{i=1}^{N} g_{i}^{o}$.

\section{$5 \quad$ Bit Energy Efficiency}

Based on the optimal transmit power and transmission time expressions derived in Section 4, the minimum expected energy consumption for transmitting one information bit in a DS-CDMA based WSN, termed bit-energy efficiency (BEE) of the network, can be studied analytically. To proceed with our analysis, we focus our attention on a homogeneous clock-driven WSN, i.e., we take $\alpha_{\text {ciri }}=$ $\alpha_{c i r}$ and $\gamma_{i}=\gamma$ for all $i$. This assumption is reasonable because BEE is typically a device-independent metric of system performance that is specified under the assumption of homogeneity. 
Initialization: For $i=1, \ldots, N, A_{i}=\frac{\delta B_{i} \gamma_{i}}{W}, g_{i}^{\text {upp }}=\delta h_{i} P_{\max }$, and $g_{i}^{\text {low }}=\frac{\delta B_{i} \gamma_{i}}{\delta B_{i} \gamma_{i}+W T_{i}^{l i m i t}}$ $K=\sum_{i=1}^{N} \delta^{-1} h_{i}^{-1} A_{i}, t_{1}=1, t_{2}=\frac{\delta P_{\max } h_{\Sigma}}{1+\delta P_{\max } h_{\Sigma}}$

$\mathbf{V}=\{1, \ldots, N\}, \mathbf{U}=\emptyset$, and flag-continue $=$ TRUE

For all $i \in \mathbf{V}-\mathbf{U}$

$$
f_{i}^{(1)}\left(t_{1}, \mathbf{U}\right)=\frac{t_{1} \sqrt{\alpha_{i} A_{i}}}{\sqrt{K}+\sum_{j \in \mathbf{V}-\mathbf{U}} \sqrt{\alpha_{j} A_{j}}}, f_{i}^{(2)}\left(t_{2}, \mathbf{U}\right)=\frac{t_{2} \sqrt{\alpha_{i} A_{i}}}{\sum_{j \in \mathbf{V}-\mathbf{U}} \sqrt{\alpha_{j} A_{j}}}
$$

End for

$m=1 / /$ start with case 1

Iteration: $\quad$ While flag-continue $=$ TRUE, do

$$
\text { flag-continue }=\text { FALSE }
$$

For all $i \in \mathbf{V}-\mathbf{U}$, set $g_{u i}^{o}=f_{i}^{(m)}\left(t_{m}, \mathbf{U}\right)$

For all $i \in \mathbf{V}-\mathbf{U}$, do

If $g_{u i}^{o}>g_{i}^{u p p}$,

Set $g_{i}^{o}=g_{i}^{u p p}$

$\mathbf{U}=\mathbf{U} \cup\{i\}$

flag-continue $=$ TRUE

Else if $g_{u i}^{o}<g_{i}^{\text {low }}$,

End if-else

Set $g_{i}^{o}=g_{i}^{\text {low }}, \mathbf{U}=\mathbf{U} \cup\{i\}$, and flag-continue $=$ TRUE

End for

Update $t_{m}$ :

If $m=1, t_{1}=1-\sum_{i \in \mathbf{U}} g_{i}^{o}$

Else, $t_{2}=\frac{\delta P_{\max } h_{\Sigma}}{1+\delta P_{\max } h_{\Sigma}}-\sum_{j \in \mathbf{U}} g_{j}^{o}$

Update $f_{i}^{(m)}\left(t_{m}, \mathbf{U}\right)$ as in the initialization step

End while

If $\mathbf{U}=\mathbf{V}$, exit // no feasible solution

Else for all $i \in \mathbf{V}-\mathbf{U}$, set $g_{i}^{o}=g_{u i}^{o}$

If $\left(m==1 \& \& \sum_{i=1}^{N} g_{i}^{o}<\frac{\delta P_{\max } h_{\Sigma}}{1+\delta P_{\max } h_{\Sigma}}\right)$ or $\left(m==2 \& \& \sum_{i=1}^{N} g_{i}^{o}=\frac{\delta P_{\max } h_{\Sigma}}{1+\delta P_{\max } h_{\Sigma}}\right)$ output $\left(g_{1}^{o}, \ldots, g_{N}^{o}\right)$ and exit

Else // case 2

Set $\mathbf{U}=\emptyset$, flag-continue $=$ TRUE, $m=2$, and go to Iteration

Table 1: Pseudo-code for computing the optimal solution for transmit power and time. 
A well-designed WSN should not be operated at the boundary of its capacity, i.e., the load of the traffic should be reasonable compared with the network capacity so that the optimal transmit power and time allocation are located within the polyhedron depicted by the constraints of (5). Hence, we further assume that the considered WSN is well designed in the above sense. Considering (43), the optimal power index of node $i$ is given by

$$
g_{i}^{o}=\frac{\sqrt{\alpha_{c i r} A_{i}}}{\sqrt{K}+\sum_{i=1}^{N} \sqrt{\alpha_{c i r} A_{i}}}, \quad i=1, \ldots, N
$$

Substituting (53) into (26) and (31), we obtain simplified closed-form expressions for the optimal transmit power and time:

$$
\begin{aligned}
P_{t i}^{o} & =\frac{\sqrt{\alpha B_{i}}}{h_{i} \sqrt{\delta \sum_{j=1}^{N} h_{j}^{-1} B_{j}}} \\
T_{i}^{o} & =\frac{\delta B_{i} \gamma}{W g_{i}^{o}}\left(1-g_{i}^{o}\right) \simeq \frac{\delta B_{i} \gamma\left(\sqrt{\delta^{-1} \sum_{j=1}^{N} h_{j}^{-1} B_{j}}+\sum_{j=1}^{N} \sqrt{\alpha_{c i r} B_{j}}\right)}{W \sqrt{\alpha_{c i r} B_{i}}} .
\end{aligned}
$$

Substituting (54) and (55) into (4), the minimum energy required for the transmission of $\sum_{i}^{N} B_{i}$ bits in a given transmission cycle is given by

$$
\begin{aligned}
E_{\text {total }}^{\min } & =\frac{1}{\eta} \sum_{i=1}^{N}\left(\frac{\sqrt{\alpha_{c i r} B_{i}}}{h_{i} \sqrt{\delta \sum_{j=1}^{N} h_{j}^{-1} B_{j}}}+\alpha_{c i r}\right) \frac{B_{i} \gamma\left(\sqrt{\delta \sum_{j=1}^{N} h_{j}^{-1} B_{j}}+\sum_{j=1}^{N} \delta \sqrt{\alpha_{c i r} B_{j}}\right)}{W \sqrt{\alpha_{c i r} B_{i}}} \\
& =\frac{\gamma}{W \eta}\left(\sum_{i=1}^{N} B_{i} h_{i}^{-1}+2 \sqrt{\alpha_{c i r}} \delta \sum_{i=1}^{N} \sqrt{\sum_{j=1}^{N} h_{j}^{-1} B_{i} B_{j}}+\alpha_{c i r} \delta \sum_{i=1}^{N} \sum_{j=1}^{N} \sqrt{B_{i} B_{j}}\right) .
\end{aligned}
$$

Suppose that $B_{i}$ and $h_{i}, i=1, \ldots, N$, are arbitrarily defined random variables. Taking the expectation of (56) with respect to $B_{i}$ and $h_{i}$ gives $\mathrm{E}\left\{E_{\text {total }}^{\min }\right\}$; the minimum expected energy consumption in one transmission cycle. In general, $\mathrm{E}\left\{E_{\text {total }}^{\min }\right\}$ can not be expressed in a closed form. However, as stated in Proposition 6, a tight upper bound can be obtained using the first-order moment $\mathrm{E}\{\mathbf{B}\}$ and the covariance matrix $\mathrm{E}\left\{\mathbf{B}^{T} \mathbf{B}\right\}$.

\section{Proposition 6:}

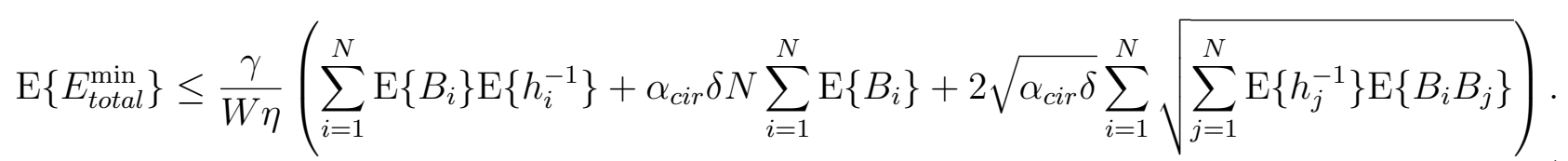

Proof: Because the geometric average of a sequence of nonnegative numbers can not be larger than their arithmetic average, we have

$$
\begin{aligned}
\mathrm{E}\left\{\alpha_{c i r} \delta \sum_{i=1}^{N} \sum_{j=1}^{N} \sqrt{B_{i} B_{j}}\right\} & =\alpha_{c i r} \delta \sum_{i=1}^{N} \sum_{j=1}^{N} \mathrm{E}\left\{\sqrt{B_{i} B_{j}}\right\} \\
& \leq \alpha_{c i r} \delta \sum_{i=1}^{N} \sum_{j=1}^{N} \frac{\mathrm{E}\left\{B_{i}\right\}+\mathrm{E}\left\{B_{j}\right\}}{2} \\
& =\alpha_{c i r} \delta N \sum_{i=1}^{N} \mathrm{E}\left\{B_{i}\right\}
\end{aligned}
$$


In addition, because $\sqrt{x}$ is a concave function for $x \geq 0$, according to Jensen's inequality, $\mathrm{E}\{\sqrt{x}\} \leq$ $\sqrt{\mathrm{E}\{x\}}$. Therefore,

$$
\begin{aligned}
\mathrm{E}\left\{\sqrt{\left.\sum_{j=1}^{N} h_{j}^{-1} B_{i} B_{j}\right\}}\right. & \leq \sqrt{\mathrm{E}\left\{\sum_{j=1}^{N} h_{j}^{-1} B_{i} B_{j}\right\}} \\
& =\sqrt{\sum_{j=1}^{N} \mathrm{E}\left\{h_{j}^{-1}\right\} \mathrm{E}\left\{B_{i} B_{j}\right\}} .
\end{aligned}
$$

where we assume that the channel gain $h_{i}$ is independent of $B_{i}$. Substituting (58) and (59) into the expectation of (56), (57) follows.

If (57) is convergent, an upper bound on the BEE is obtained by dividing (57) over the average number of bits transmitted in one transmission cycle, i.e.,

$\mathrm{BEE} \leq \frac{\gamma}{W \eta \sum_{i=1}^{N} \mathrm{E}\left\{B_{i}\right\}}\left(\sum_{i=1}^{N} \mathrm{E}\left\{B_{i}\right\} \mathrm{E}\left\{h_{i}^{-1}\right\}+\alpha_{c i r} \delta N \sum_{i=1}^{N} \mathrm{E}\left\{B_{i}\right\}+2 \sqrt{\alpha_{c i r} \delta} \sum_{i=1}^{N} \sqrt{\sum_{j=1}^{N} \mathrm{E}\left\{h_{j}^{-1}\right\} \mathrm{E}\left\{B_{i} B_{j}\right\}}\right.$.

Further simplification of this upper bound as well as closed-form expressions of the BEE can be obtained for special cases of $\mathbf{B}$, as described next.

\subsection{Sensor Nodes with Independent and Identically Distributed Traffic}

If $B_{i}$ 's are i.i.d. random variables and $N$ is large, it can be shown that (60) can be further simplified to a traffic-distribution-independent asymptotic upper bound

$$
\mathrm{BEE}^{i i d} \leq \frac{\gamma}{W \eta}\left(\frac{\mathrm{E}\{G\}}{N}+\alpha_{c i r} \delta N+2 \sqrt{\alpha_{c i r} \delta \mathrm{E}\{G\}}\right)
$$

where $G \stackrel{\text { def }}{=} \sum_{i=1}^{N} h_{i}^{-1}$ is the sum of the inverse of channel gains.

\subsection{Sensor Nodes with Fully Correlated Traffic}

In many WSN scenarios, the data captured and transmitted by various sensors are highly correlated. As an extreme case, suppose the numbers of bits transmitted by various sensor nodes in the same cycle are identical although they may vary in consecutive cycles, i.e., $B_{1}=B_{2}=\ldots=B_{N}$ with $E\left(B_{i}\right)=B$. In this case, the BEE is given by

$$
\mathrm{BEE}^{F C}=\frac{\gamma}{W \eta}\left(\frac{\mathrm{E}\{G\}}{N}+\alpha_{c i r} \delta N+2 \sqrt{\alpha_{c i r} \delta} \mathrm{E}\{\sqrt{G}\}\right) .
$$

A special yet widely used case is a WSN employing fixed-length coding, i.e., the number of bits transmitted by each sensor node in each cycle is a constant $B$.

\section{$6 \quad$ Numerical Investigations}

In this section, we verify the accuracy of our analysis by comparing the analytical results obtained in Section IV with those of the numerical algorithm presented in Section III. The effect of relaxing the constraints and that of other approximations made in our analysis are also investigated. 


\subsection{System Settings}

We consider a $20 m \times 20 m$ square sensing field, as shown in Figure 2, over which $N$ homogeneous sensors are distributed uniformly. The sink node is located at $(D, 0)$. For each sensor node, the power amplifier energy efficiency is set to $\eta=0.9$. The network is clock-driven and in every cycle of 1 second, all $N$ sensors transmit their data simultaneously using DS-CDMA. A rectangular spreading chip is assumed, i.e. $\delta=\frac{2}{3}$. The threshold of the received SINR is 4 for all nodes. Each transmission must be completed within $T_{i}^{\text {limit }}=1$ second. The spread spectrum bandwidth is $W=1 \mathrm{MHz}$ and the single-sided power spectrum density of AWGN is $N_{0}=10^{-15} \mathrm{~W} / \mathrm{Hz}$. For sensor node $i$, the channel gain is given by

$$
h_{i}=L\left(d_{0}\right)\left(\frac{d_{i}}{d_{0}}\right)^{-\mu} Y_{i}\left(X_{I i}^{2}+X_{Q i}^{2}\right),
$$

where $L\left(d_{0}\right)=\frac{G_{t} G_{r} \lambda^{2}}{16 \pi^{2} d_{0}^{2}}$ is the path loss of the close-in distance $d_{0}, G_{t}$ and $G_{r}$ are the antenna gains of the transmitter and the receiver, respectively, and $\lambda$ is the wavelength of the carrier. We take $d_{0}=10$ meters and $G_{t} G_{r}=1$. We also set the carrier frequency to $2.4 \mathrm{GHz}$. Let $d_{i}$ be the distance between node $i$ and the sink. The parameters $Y_{i}, i=1, \ldots, N$, are i.i.d. lognormally distributed random variables with standard deviation $7 \mathrm{~dB}$. They account for the effect of shadowing. Moreover, $X_{I i}$ and $X_{Q i}$ are the real and the imaginary parts of a Rayleigh fading channel gain, which follows a Gaussian distribution of mean zero and variance $\frac{1}{2}$. Finally, $\mu$ is the path loss exponent and is assumed to be 2 in our system, i.e., we consider a free-space loss model.

\subsection{Numerical Results}

In Figures 3 and 4, we depict the results obtained from the GP-based numerical algorithm and from the analytical algorithm proposed in Sections 3 and 4, respectively. For a given cycle, the channel gain of each node is generated according to (63). Both numerical and analytical algorithms are applied to calculate the optimal transmit power and transmission time for each node. The traffic generated by different nodes in each cycle is i.i.d. with a Poisson distribution of mean 100 bits. Although other, more realistic traffic models can be used in the simulations, this will have no impact on the qualitative (relative) performance of various optimization approaches. Figures 3 and 4 depict, respectively, the energy consumption and the average sensor transmission time in each cycle for 10 consecutive cycles. To illustrate the benefits of jointly optimizing transmit power and time, we also include in Figure 3 the performance of a "fixed-transmission-time" strategy [3], whereby the transmission time for each sensor is set to the delay constraint $(1 s)$ and the transmit power is determined using (26). It can be observed that despite the approximate nature of our closed-form solution, this solution is almost indistinguishable from the GP-based numerical solution. This accuracy can be explained by noting that for a typical CDMA-based WSN with a low data transmission rate, large spread spectrum bandwidth, and a small received SINR requirement, $g_{i} \ll 1$.

It should be noted, however, that the relaxation of the constraint on $g_{i}$ from (28) into (30) may result in some nodes having optimal transmit powers greater than $P_{\max }$. Such nodes will obviously have to use $P_{\max }$ as their transmit power. Fortunately, this capping of power will only impact the the signal quality of such nodes (the SINR of other nodes will actually improve).

In Figures 5 and 6, we study the severity of violating the $P_{\max }$ constraint as a function of $P_{\max }$. We use two metrics for this purpose: violation rate and violation degree. The violation rate is defined as the average percentage of sensors in a cycle whose optimal transmit powers exceed $P_{\max }$. The violation degree is defined as the average power surplus over $P_{\max }$ required by those violating sensors. This value is normalized by $P_{\max }$. It is observed that for a wide range of $N$ values (20 to 100), even 
under a tight power constraint of $10 \mathrm{~mW}$, only a small percentage of sensors $(\approx 5 \%)$ violate the $P_{\max }$ constraint to a degree of $25 \%$. Effectively, this says that in each transmission cycle, about $5 \%$ of the information bits are received at the sink below their SINR threshold with a normalized deficit of 0.25. Taking advantage of the rich data redundancy possessed by a WSN, the $5 \%$ data loss can be easily compensated for by other data transmitted from neighboring nodes. Using a more practical value for $P_{\max }=100 \mathrm{~mW}[11]$, the violation rate and degree are reduced to below $0.2 \%$ and $20 \%$, respectively (over various values of $N$ ).

In Figures 7 through 10, we study the BEE performance under various traffic scenarios. Figure 7 depicts the BEE versus $N$ for the case of fully correlated traffic. The theoretical values (obtained from (62)) are compared with those from simulations where $B_{i}$ is assumed to have a Poisson distribution with mean 100. In the simulations, the GP-based numerical algorithm is employed to determine the optimal transmit power and time in each transmission cycle. The figure shows that (62) accurately captures the BEE performance of a WSN. The case of i.i.d. traffic is considered in Figures 8-10, where the BEE is plotted as a function of the circuit power consumption $(\alpha)$, the remote node distance $(D)$, and $N$, respectively. In these figures, we contrast the distribution-independent theoretical upper bound on the BEE (given in (61)) with three simulation-based BEE values that correspond to three different traffic distributions. The theoretical bound is found to be sufficiently tight. The simulation results also show that the BEE decreases with the increase in the variance of the traffic (compare the results for the cases $B_{i} \sim$ uniform $(50,150)$ and $B_{i} \sim$ uniform $\left.(20,180)\right)$. This can be attributed, in part, to the nonlinearity of $E_{\text {total }}^{\min }$, given in (56). For example, consider the term $B_{1} B_{2}$ in $(56)$. Under the constraint that $B_{1}+B_{2}=2 B$, where $B$ is a constant, we have $B_{1} B_{2}=-B_{1}^{2}+2 B B_{1}$ where $0 \leq B_{1} \leq 2 B$. It is easy to see that $B_{1} B_{2}$ is a concave function for $0 \leq B_{1} \leq 2 B$, with its maximum value attained at $B_{1}=B_{2}=B$. This says that the function $B_{1} B_{2}$ is a mono-decrease function of the absolute difference between $B_{1}$ and $B_{2}$. Similarly, for a traffic distribution with a larger variation, the expected absolute difference between $B_{i}$ and $B_{j}$ in (56) will be larger, leading to a smaller product of $B_{i} B_{j}$, hence resulting in a smaller $E_{t o t a l}^{\min }$ and BEE.

\section{Summary}

In this paper, we studied the problem of jointly optimizing the transmission powers and times of sensor nodes in a DS-CDMA WSN. The optimization was carried out for the purpose of minimizing the total energy consumption in the network. A comprehensive energy model was used, which accounts for both the transmit power consumption and the circuit energy consumption. The problem was formulated as a non-convex geometric program. In general, the non-convexity of the objective function and the constraints in such problems makes it quite challenging to obtain closed-form solutions. We first showed that the formulation can be transformed into a convex geometric program for which fast computational algorithms, such as the Interior Point Method, are applicable. Then, by exploiting the special structure of the underlying formulation, we derived a closed-form tight approximation for the optimal transmit powers and transmission times. To the best of our knowledge, this is the first closed-form analytical treatment of the subject. Our closed-form solution is based on decoupling the optimization problem into two sequential sub-problems. First, we optimize the transmit powers, treating the transmission times as parameters. As a result of this step, the optimal powers are expressed as functions of the transmission times. In the second sub-problem, we optimize the transmission times. We showed that the first sub-problem is a linear program, while the second one can be well approximated as a convex optimization problem. Taking advantage of our closedform results, we further studied the bit energy efficiency for CDMA-based WSNs under various 
traffic scenarios. We obtained closed-form expressions and bounds for the BEE. The goodness of our solutions were verified through comparisons with simulation-based numerical results. These comparisons indicate that the closed-form expressions are extremely accurate, and can therefore be used as a basis for determining the optimal transmit power and times in a WSN. Our future work will focus on using such results in the design of protocols for dynamic adjustment of the powers and times.

\section{References}

[1] I. F. Akyildiz, W. Su, Y. Sankarasubramaniam, and E. Cayirci, "A survey on sensor networks," IEEE Communications Magazine, pp. 102-114, Aug. 2002.

[2] J. M. Kahn, R. H. Katz, and K. S. Pister, "Next century challenges: mobile networking for smart dust," in Proc. ACM/IEEE MobiCom 99, pp. 271-278, 1999.

[3] R. Berry and R. Gallager, "Communication over fading channels with delay constraints," IEEE Trans. on Inform. Theory, vol. 48, no. 5, pp. 1135-1149, May 2002.

[4] E. Uysal-Biyikoglu, B. Prabhakar, and A. E. Gamal, "Energy-efficient packet transmission over a wireless link," IEEE/ACM Transactions on Networking, vol. 10, no. 4, pp. 487-499, Aug. 2002.

[5] A. E. Gamal, C. Nair, B. Prabhakar, E. Uysal-Biyikoglu, and S. Zahedi, "Energy-efficient scheduling of packet transmissions over wireless networks," in Proc. INFOCOM 2002, vol. 3, pp. 1773-1783, June 2002 .

[6] D. Rajan, A. Sabharwal, and B. Aazhang, "Delay bounded packet scheduling of bursty traffic over wireless channels," IEEE Transactions on Information Theory, vol. 50, no. 1, pp. 125-144, Jan. 2004.

[7] M. A. Khojastepour and A. Sabharwal, "Delay-constrained scheduling: power efficiency, filter design, and bounds," in Proc. INFOCOM 2004, March 2004.

[8] H. Dai, L. Xiao, and Q. Zhou, "On cooperative MIMO transmission strategies in wireless sensor networks with mobile agents," invited paper, to appear in 2004 International Conference on Computing, Communications and Control Technologies, Aug. 2004.

[9] S. Cui, A. J. Goldsmith, and A. Bahai, "Modulation optimization under energy constraints," in Proc. IEEE ICC'03, vol. 4, pp. 2805-2811, May 2003.

[10] S. Cui, A.J. Goldsmith, and A. Bahai, "Energy-constrained modulation optimization for coded systems," in Proc. IEEE Globecom'03, vol. 1, pp. 372-376, Dec. 2003.

[11] S. Cui, A.J. Goldsmith, and A. Bahai, "Energy-constrained modulation optimization," to appear in IEEE Transactions on Wireless Communications, 2004.

[12] S. Cui, A.J. Goldsmith, and A. Bahai, "Joint modulation and multiple access optimization under energy constraints," to appear in IEEE Globecom'04, Nov 2004.

[13] C.H. Liu and H.H. Asada, "A source coding and modulation method for power saving and interference reduction in DS-CDMA sensor network systems," in Proc. American Control Conference 2002, vol. 4, pp. 3003-3008, May 2002. 
[14] P. Venkitasubramaniam, S. Adireddy, and L. Tong, "Sensor networks with mobile access: optimal random access and coding," IEEE Journal on Selected Areas in Communications, vol. 22, no. 6, pp. 1058-1068, Aug. 2004.

[15] A. Sampath, P.S. Kumar, and J.M. Holtzman, "Power control and resource management for a multimedia CDMA wireless system," in Proc. IEEE Int. Symp. Personal, Indoor, Mobile Radio Communications (PIMRC'95), vol. 1, pp. 21-25, 1995.

[16] S. J. Lee and D. K. Sung, "Capacity evaluation for DS-CDMA systems with multi-class on/off traffic," IEEE Commun. Letters, vol. 2, no. 6, pp. 153-155, June 1998.

[17] W. B. Heinzelman, A. P. Chandrakasan, and H. Balakrishnan, "An application-specific protocol architecture for wireless microsensor networks," IEEE Transactions on Wireless Communications, vol. 1, no. 4, pp. 660-670, Oct. 2002.

[18] S. Bandyopadhyay and E. J. Coyle, "An energy efficient hierarchical clustering algorithm for wireless sensor networks," in Proc. IEEE INFOCOM 2003, vol. 3, pp. 1713-1723, Mar. 2003.

[19] J. Pan, Y.T. Hou, L. Cai, Y. Shi, and S. X. Shen, "Topology control for wireless sensor networks," in Proc. ACM MobiCom 2003, pp. 286-299, 2003.

[20] T.H. Lee, The Design of CMOS Radio-Frequency Integrated Circuits. Cambridge Univ. Press, Cambridge, U.K., 1998.

[21] S. Boyd and L. Vandenberghe, Convex Optimization, Cambridge Univ. Press, Cambridge, U.K., 2004.

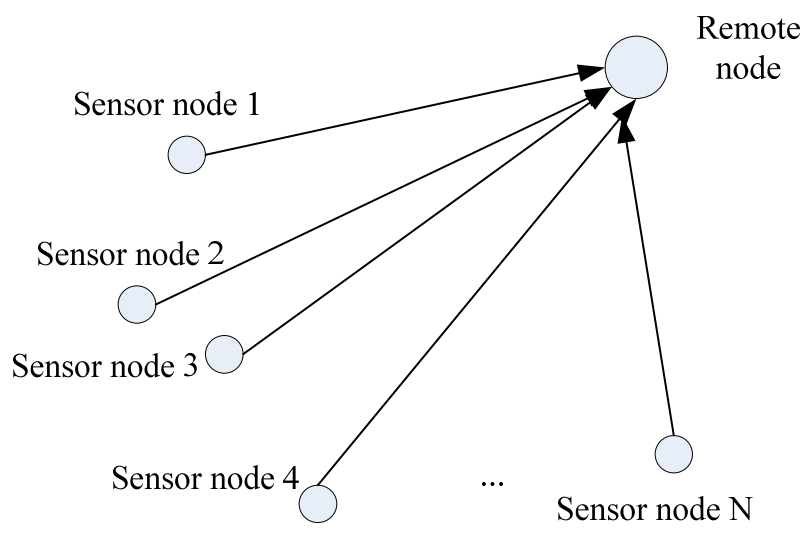

Figure 1: System model. 


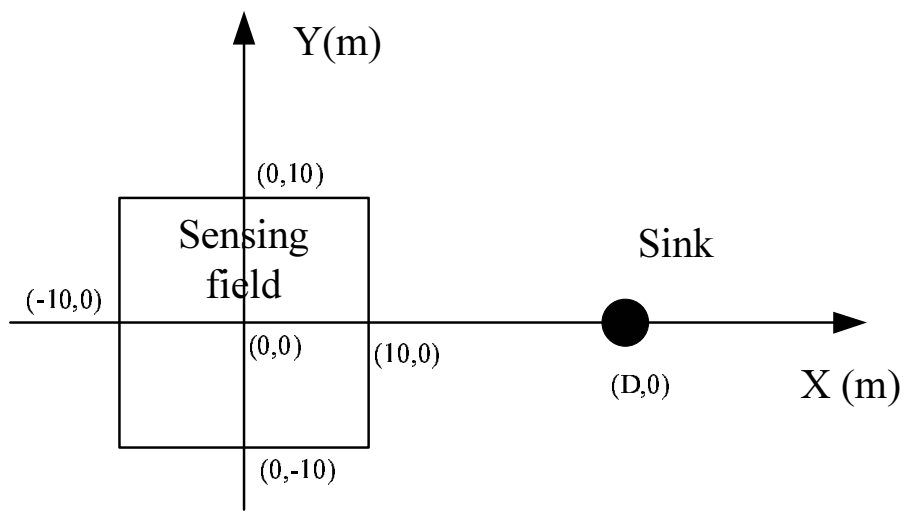

Figure 2: Sensing field used in the numerical examples.

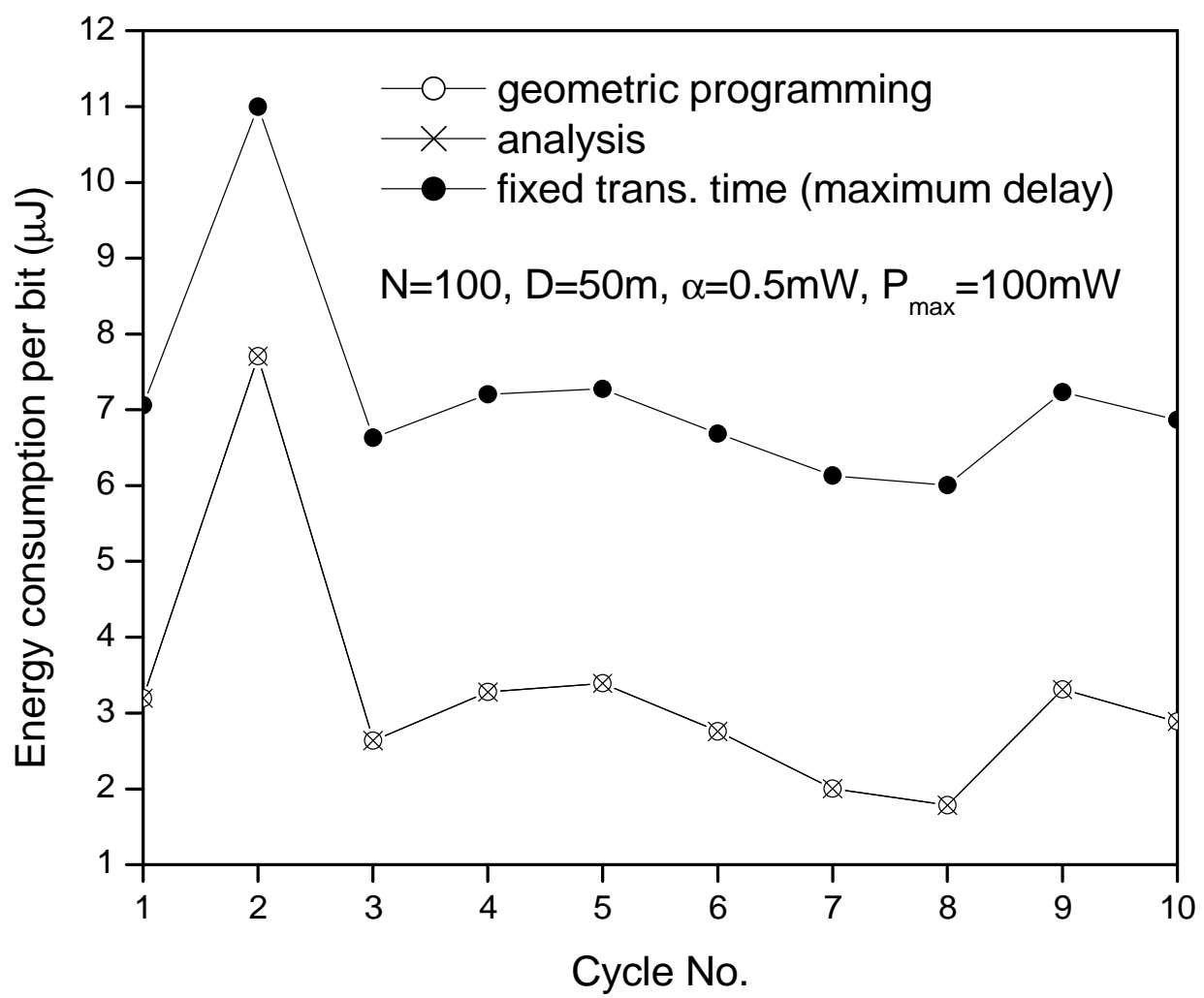

Figure 3: Trace of energy consumption per bit for ten successive cycles $(N=100)$. 


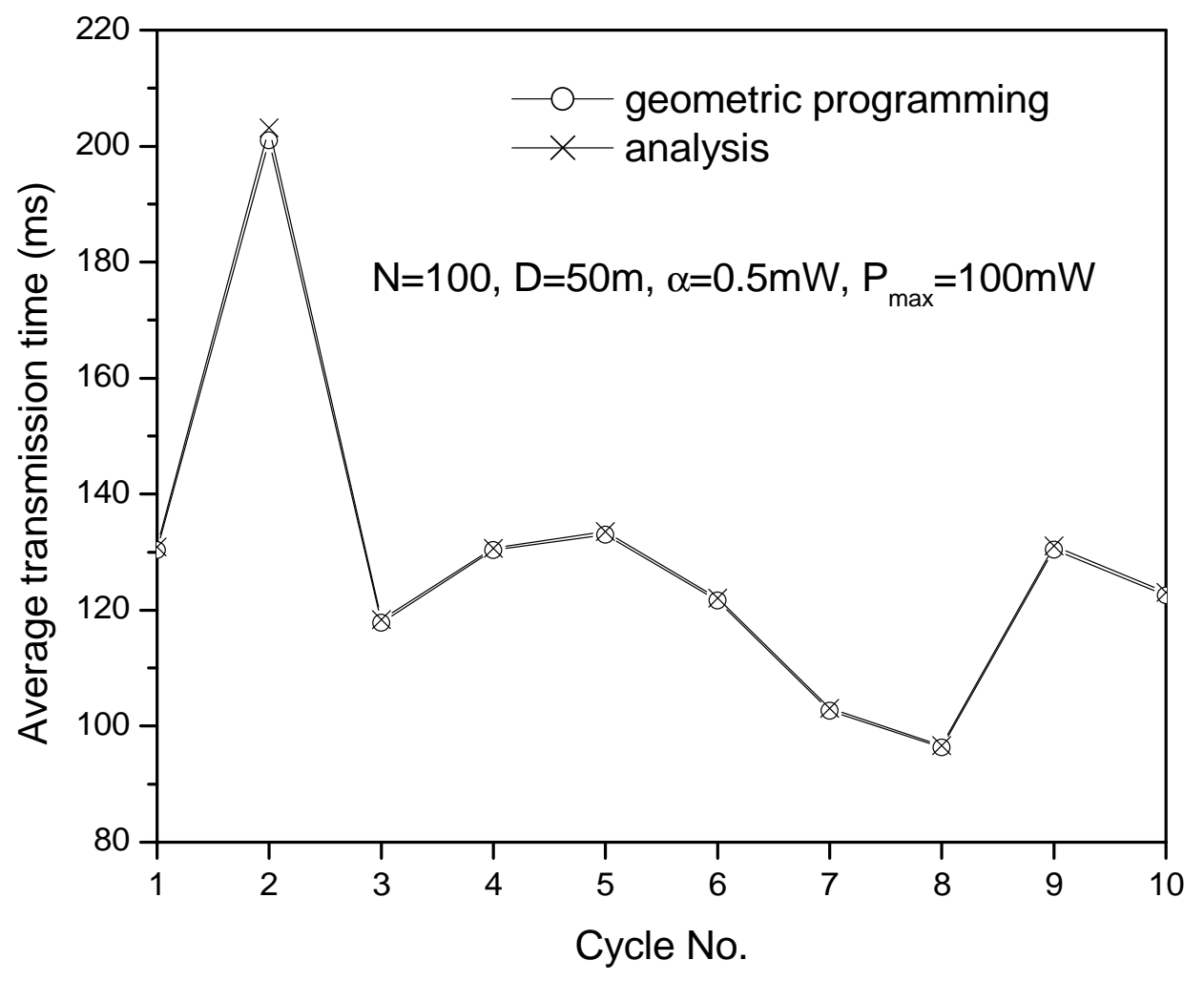

Figure 4: Trace of average sensor transmission time in ten successive cycles $(N=100)$.

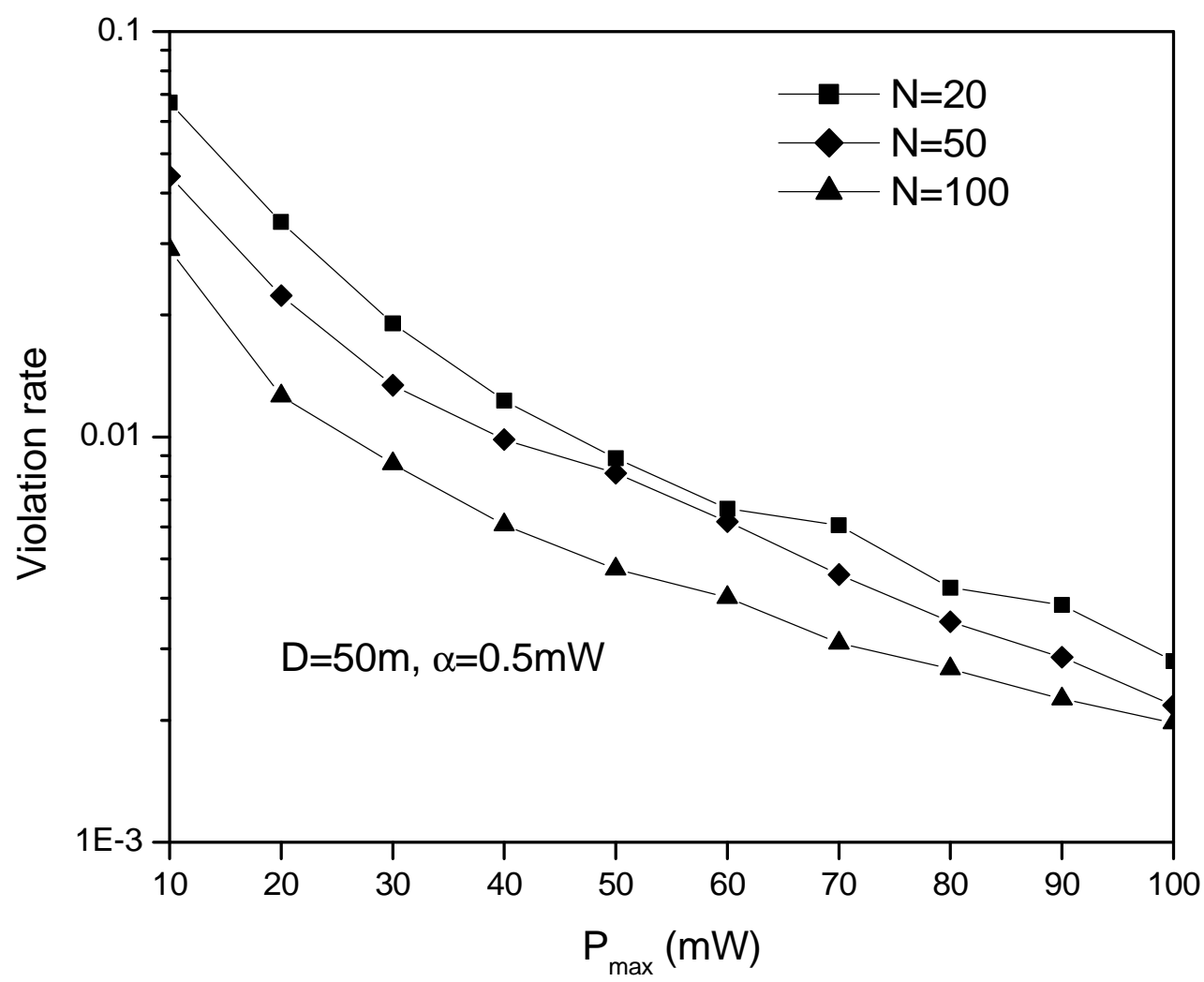

Figure 5: Violation rate of transmission power constraint vs. $P_{\max }$. 


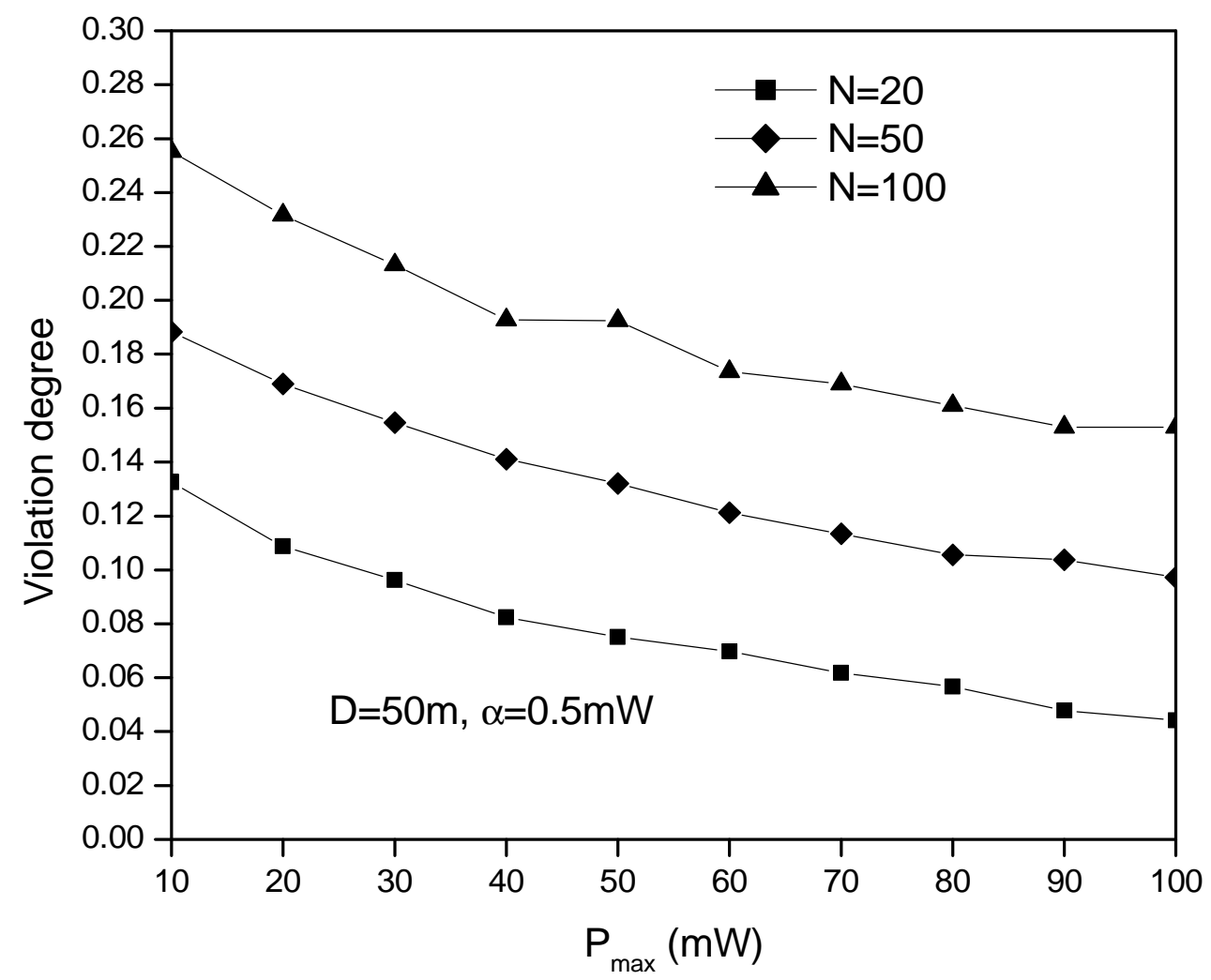

Figure 6: Violation degree of transmission power constraint vs. $P_{\max }$.

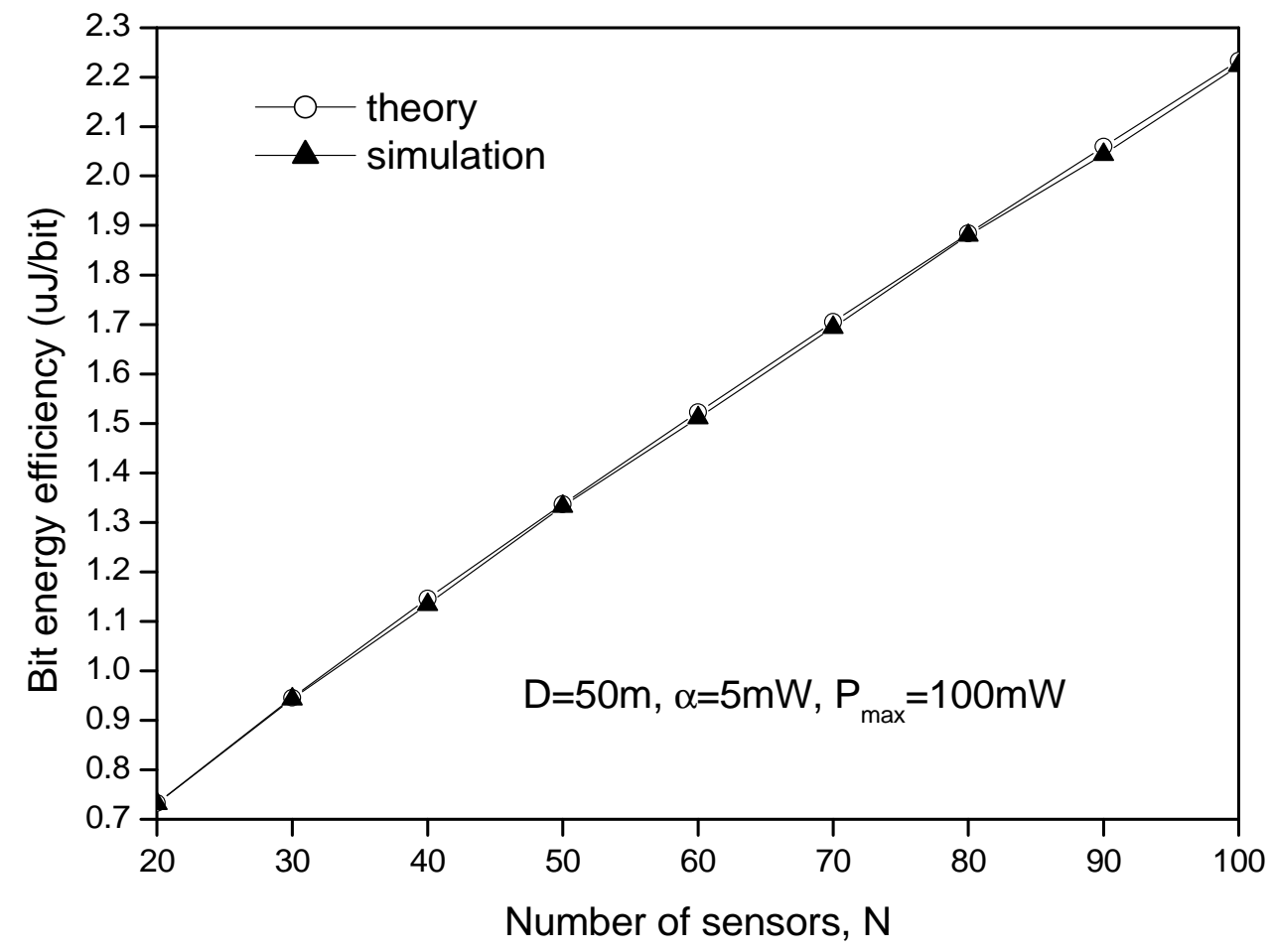

Figure 7: Bit energy efficiency vs. number of sensors, case of fully correlated nodes. 


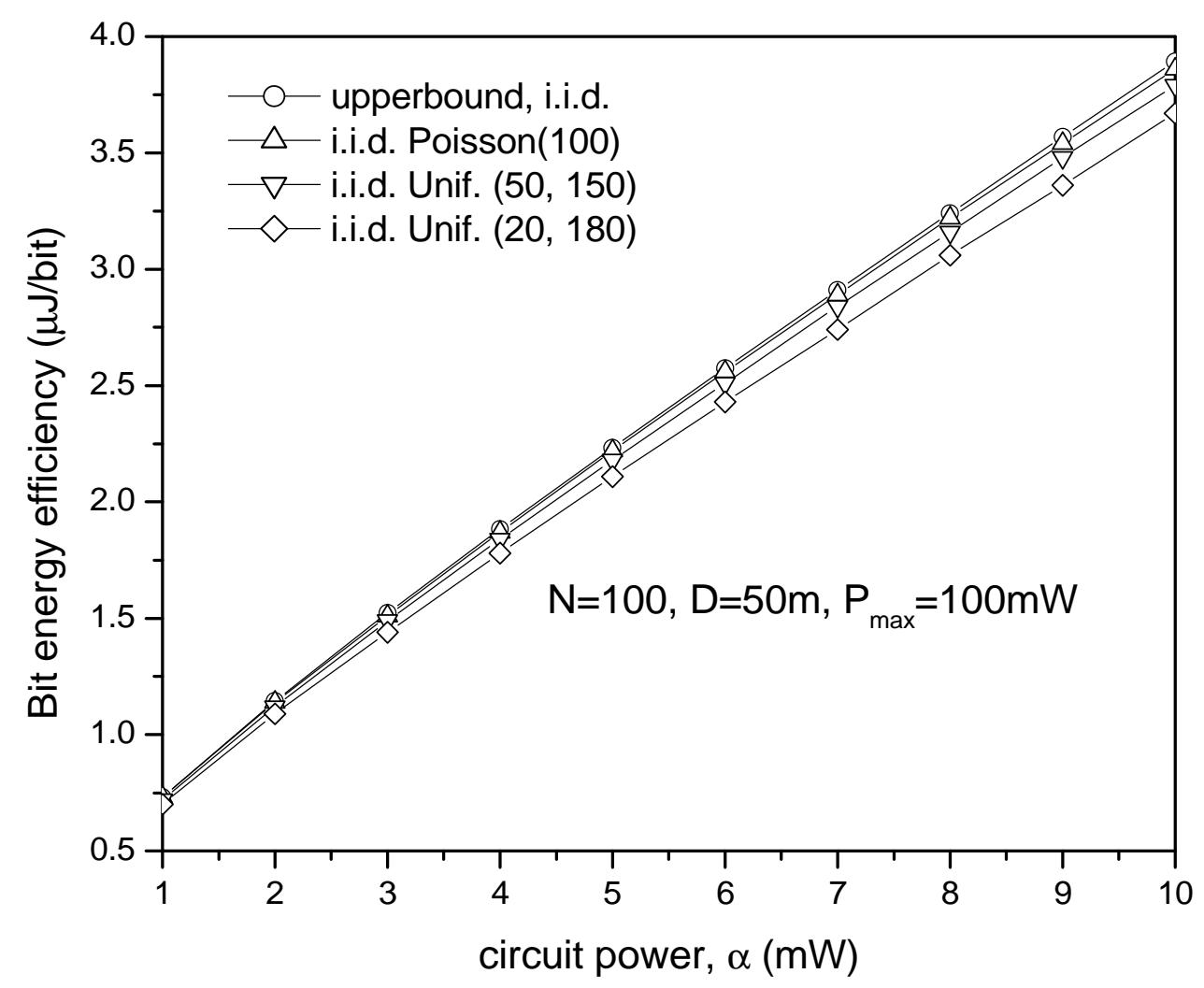

Figure 8: Bit energy efficiency vs. circuit power, case of i.i.d. nodes.

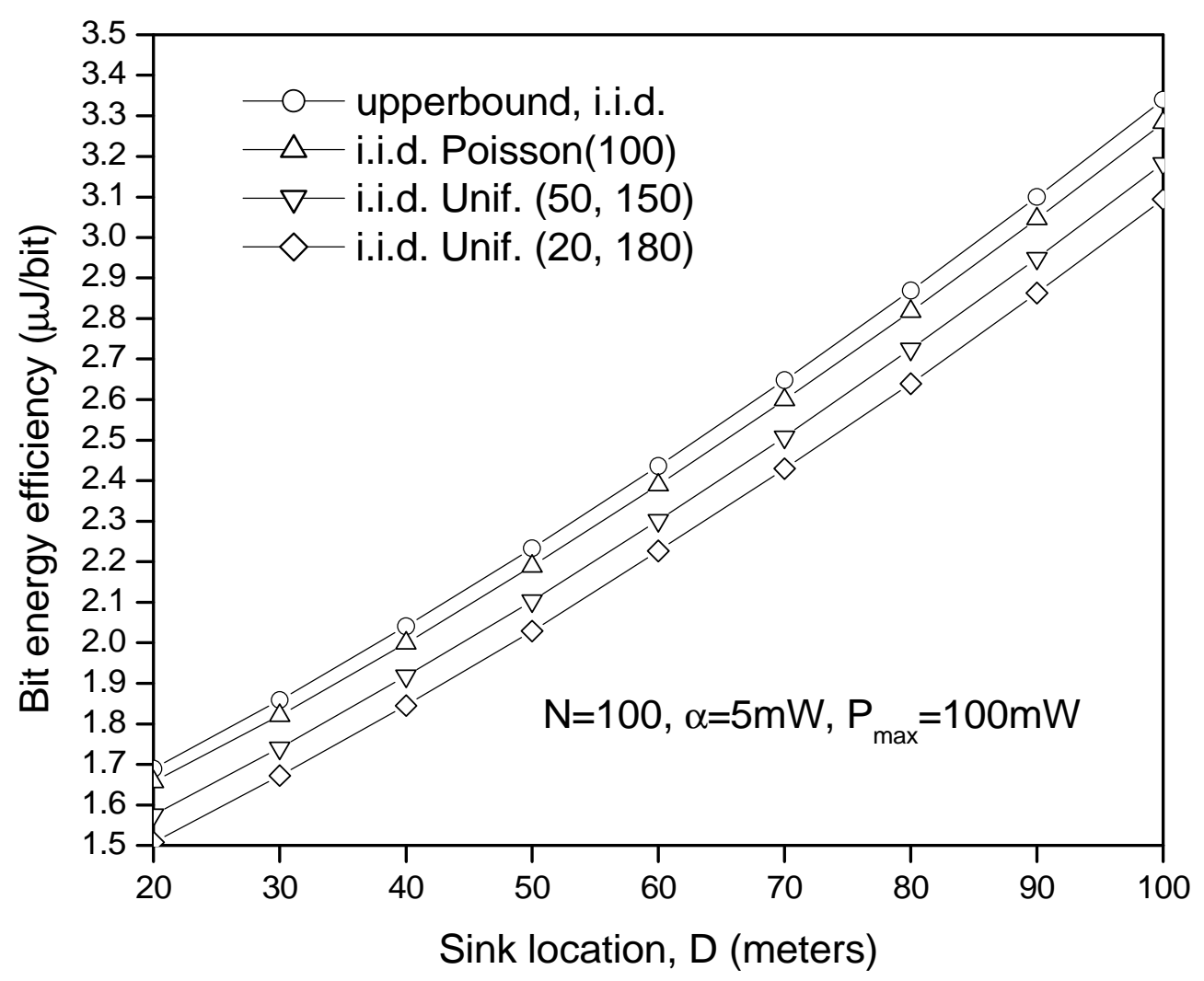

Figure 9: Bit energy efficiency vs. sink location, case of i.i.d. nodes. 


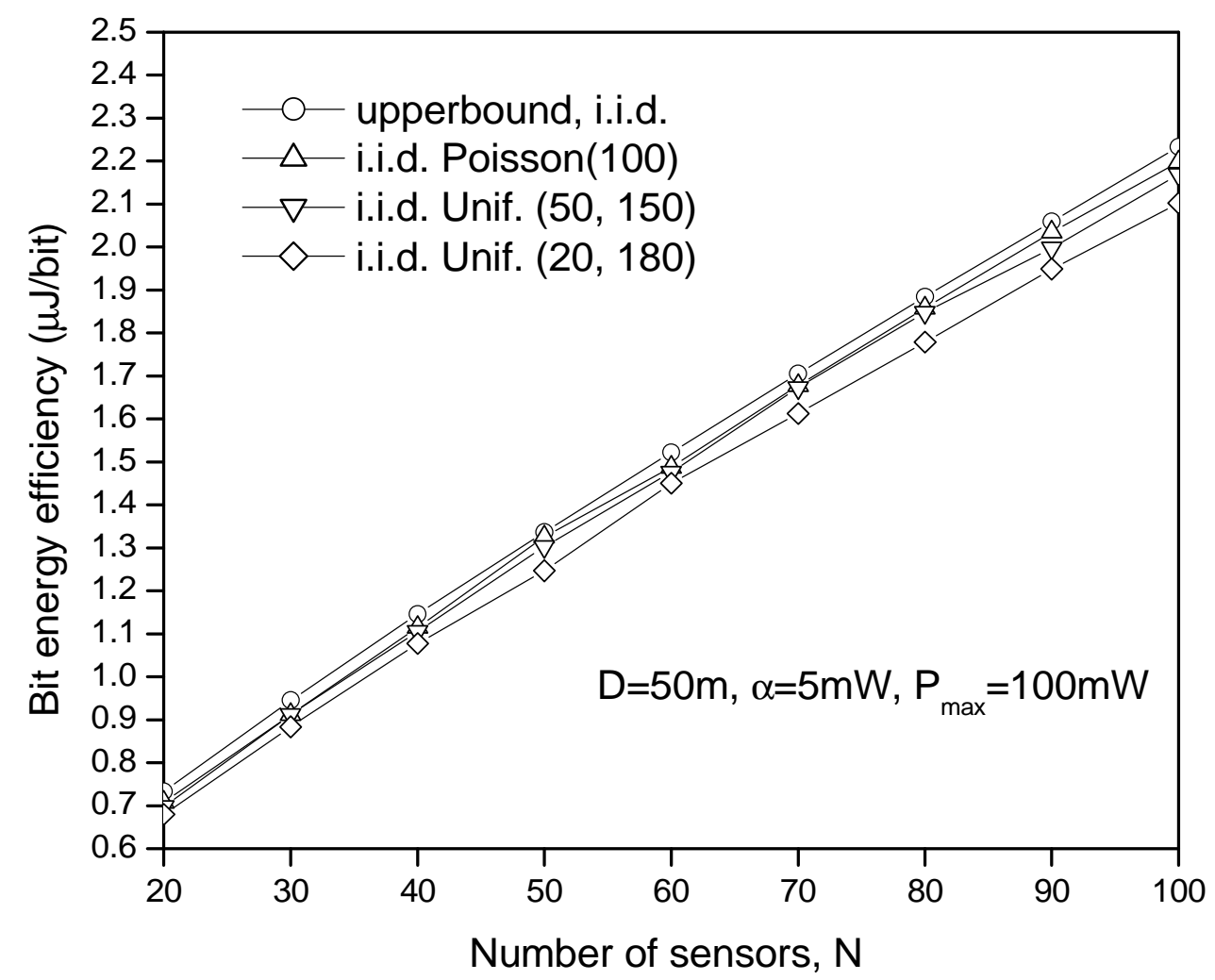

Figure 10: Bit energy efficiency vs. number of sensors, case of i.i.d. nodes. 